\title{
Mutations in TMEM43 cause autosomal dominant auditory neuropathy spectrum disorder via interaction with connexin-mediated passive conductance channels
}

Minwoo Wendy Jang ${ }^{1,2^{*}}$, Doo-Yi Oh ${ }^{3 *}$, Eunyoung $\mathrm{Yi}^{4^{*}}$, Xuezhong Liu ${ }^{5,6^{*}}$, Jie Ling ${ }^{7,8^{*}}$, Nayoung $\mathrm{Kim}^{9}$, Kushal Sharma ${ }^{4}$, Tai Young Kim ${ }^{2}$, Seungmin $\mathrm{Lee}^{3}$, Ah-Reum Kim ${ }^{3}$, Min Young Kim ${ }^{3}$, Min-A Kim ${ }^{10,11}$, Mingyu Lee ${ }^{12}$, Jin-Hee Han ${ }^{3}$, Jae Joon Han ${ }^{13}$, Hye-Rim Park ${ }^{3}$, Bong Jik Kim ${ }^{3}$, Sang-Yeon Lee ${ }^{13}$, Dong Ho $\mathrm{Woo}^{14}$, Jayoung $\mathrm{Oh}^{3}$, Soo-Jin $\mathrm{Oh}^{15}$, Tingting Du ${ }^{16}$, Ja-Won $\mathrm{Koo}^{3}$, Seung Ha Oh ${ }^{13}$, Hyun Woo Shinn ${ }^{13}$, Moon-Woo Seong ${ }^{17}$, Kyu Yup Lee ${ }^{18}$, Un-Kyung Kim ${ }^{10,11}$, Jung Bum Shin ${ }^{16}$, Shushan Sang ${ }^{7}$, Xinzhang $\mathrm{Cai}^{7}$, Lingyun $\mathrm{Mei}^{7}$, Chufeng $\mathrm{He}^{7}$, Susan H. Blanton ${ }^{5,6}$, Zheng-Yi Chen ${ }^{19}$, Hongsheng $\mathrm{Chen}^{7}$, Xianlin Liu ${ }^{7}$, Aida Nourbakhsh ${ }^{5}$, Zaohua Huang ${ }^{5}$, Woong-Yang Park ${ }^{9}$, Yong Feng ${ }^{7,20 \dagger}$, C. Justin Lee ${ }^{2 \dagger}$, Byung Yoon Choi ${ }^{3 \dagger}$

${ }^{1}$ KU-KIST Graduate School of Converging Science and Technology, Korea University, Seoul 02841, Korea

${ }^{2}$ Center for Cognition and Sociality, Institute for Basic Science (IBS), Daejeon 34141, Korea

${ }^{3}$ Department of Otorhinolaryngology, Seoul National University Bundang Hospital, Seongnam 13620, Korea

${ }^{4}$ College of Pharmacy and Natural Medicine Research Institute, Mokpo National University, Mokpo, Korea

${ }^{5}$ Department of Otolaryngology, University of Miami Miller School of Medicine, Miami, FL, USA

${ }^{6}$ Dr. John T. Macdonald Foundation Department of Human Genetics and Hussman Institute for Human Genomics, University of Miami Miller School of Medicine, Miami, FL, USA

${ }^{7}$ Department of Otolaryngology, Xiangya Hospital, Central South University, Changsha, Hunan, China

${ }^{8}$ Institute of Molecular Precision Medicine, Xiangya Hospital, Central South University, Changsha, Hunan, China

${ }^{9}$ Samsung Genome Institute, Samsung Medical Center, Seoul, Korea

${ }^{10}$ Department of Biology, College of Natural Sciences, Kyungpook National University, Daegu, 41566, Republic of Korea

${ }^{11}$ School of Life Sciences, KNU Creative BioResearch Group (BK21 plus project), Kyungpook National University, Daegu, 41566, Republic of Korea

${ }^{12}$ Department of Biomedical Sciences, Seoul National University Graduate School, Seoul, Korea ${ }^{13}$ Department of Otorhinolaryngology, Seoul National University Hospital, Seoul, Korea

${ }^{14}$ Research Center for Animal Model, Jeonbuk Department of Inhalation Research, Korea Institute of Toxicology, KRICT, Jeongeup 56212, Korea

${ }^{15}$ Convergence Research Center for Diagnosis, Treatment and Care System of Dementia, Korea Institute of Science and Technology (KIST) Seoul 02792, Korea

${ }^{16}$ Department of Neuroscience, University of Virginia, Charlottesville, Virginia 22908, USA

${ }^{17}$ Department of Laboratory Medicine, Seoul National University Hospital, Seoul National University College of Medicine, Seoul, Korea 
$42 \quad{ }^{18}$ Department of Otorhinolaryngology-Head and Neck Surgery, Kyungpook National University

43 Hospital, Kyungpook National University School of Medicine, Daegu, Korea

$44{ }^{19}$ Department of Otology and Laryngology, Harvard Medical School and Eaton-Peabody

45 Laboratory, Massachusetts Eye and Ear Infirmary, Boston 02114, USA

$46{ }^{20}$ Changsha Central Hospital, University of South China, Changsha, Hunan, China

47

48 *These authors contributed equally to this work.

49 Correspondence and requests for materials should be addressed to B.Y.C. (email:

50 choiby2010@gmail.com) or to C.J.L. (email: cj1@ibs.re.kr) or to Y.F.

51 (fengyong_hn@hotmail.com) 


\section{Abstract}

53 Genes that are primarily expressed in cochlear glia-like supporting cells (GLSs) have never been

54 clearly associated with progressive deafness. Herein, we present a novel deafness locus mapped

55 to chromosome 3p25.1 and a new auditory neuropathy spectrum disorder (ANSD) gene

56 TMEM43 mainly expressed in GLSs. We identify p.R372X of TMEM43 by linkage analysis and

57 exome sequencing in two large Asian families. The knock-in (KI) mouse with p.R372X mutation

58 recapitulates a progressive hearing loss with histological abnormalities exclusively in GLSs.

59 Mechanistically, TMEM43 interacts with Cx26 and Cx30 gap junction channels, disrupting the

60 passive conductance current in GLSs in a dominant-negative fashion when the p.R372X

61 mutation is introduced. Based on the mechanistic insights, cochlear implant was performed on

62 two patients and speech discrimination was successfully restored. Our study highlights a

63 pathological role of cochlear GLSs by identifying a novel deafness gene and its causal

64 relationship with ANSD. 
The main organ for hearing and speech discrimination is the organ of Corti of inner ear, and

66

67

68

69

70

71

72

73

74

75

76

77

78

79

80

81

82

83

84

85

86

87

its major cell types include neuron-like inner hair cells (IHCs) and outer hair cells (OHCs) and

glia-like supporting cells (GLSs). GLSs have been suggested to play an important role in

development and maintenance of auditory system ${ }^{1}$. These cells are defined as glia-like cells due

to typical glia markers such as GFAP and GLAST ${ }^{2}$. Mutations of connexin $(\mathrm{Cx})$ channels of

GLSs such as Cx26 (GJB2) ${ }^{3,4}$ and Cx30 (GJB6) ${ }^{4,5}$ are known to cause hearing impairment,

highlighting the importance of GLSs in deafness. However, the underlying molecular

mechanisms of how these gap junction channels interact with other proteins and lead to hearing

loss still remain to be explored.

Hearing loss is defined as a diminished sensitivity to the sounds normally heard ${ }^{6}$ or an inability in speech discrimination despite preserved sensitivity to sound as in auditory neuropathy spectrum disorder (ANSD). As of 2017, about 1.4 billion people worldwide are affected by hearing loss ${ }^{7}$. The causes of hearing loss include exposure to aging, ototoxic drugs, other environmental insults, and most importantly, genetic alterations which account for $60 \%$ of the hearing loss (http://hereditaryhearingloss.org). Recent advances in sequencing technologies have facilitated discovery of a genetic etiology of deafness with varying degrees and features ${ }^{8,9}$. Especially, understanding the roles and functions of novel genes in the auditory system has been made through a stepwise approach: cloning of a novel deafness gene and investigation of the function by in vitro studies and analyses on mutant animal models ${ }^{10-12}$. Over one hundred deafness genes have been identified in hair cells (http://hereditaryhearingloss.org). However, the genes in GLSs and their roles in hearing or speech discrimination, especially their contribution to ANSD still remains unknown. 


\section{Results}

\section{TMEM43 is a novel deafness gene}

We identified two large five-generation pedigrees of Chinese Han family (HN66) and Korean family (SB162), non-consanguineously segregating the adult-onset progressive ANSD in an autosomal dominant fashion (Fig. 1a and Extended Data Fig. 1a). Unlike the normal subject (\#290) in SB162, the affected subjects (\#17, \#291, \#284, \#304) from families HN66 and SB162 displayed elevated pure tone audiogram (PTA) thresholds and disproportionately lower speech discrimination score (SDS) for the PTA thresholds. Importantly, complete absence of auditory brainstem response (ABR) was noted from these affected subjects, despite the presence of either distortion-product otoacoustic emission (DPOAE) or cochlear microphonics (CM) (Fig. 1b), which are classic signs of ANSD. CM measurement was not performed from Subjects \#17 and \#36. These typical symptoms did not begin to appear in a full extent until the age of thirties. The youngest subject (\#36 from HN66, a 16-year-old female) showed a significant elevation of ABR threshold as high as $80 \mathrm{~dB}$, while DPOAE response of \#36 was completely preserved (Fig. 1b).

This suggests that phenotype for \#36 can be interpreted as the stage of transition to canonical ANSD.

Stepwise genetic analysis was carried out to identify the pathogenic variants of families HN66 and SB162, separately (Fig. 2a). To identify the chromosomal locus of ANSD in the two families, we conducted a whole-genome linkage-scan on 9 subjects ( 5 affected and 4 unaffected) in HN66 and 18 subjects (10 affected and 8 unaffected) in SB162 (Extended Data Fig. 1c). As a result, we identified a candidate region on chromosome $3: 13,165,401-22,769,511$ with the highest parametric $\log _{10}$ Odds (LOD) value of 2.4 across the entire chromosomes in HN66 (Fig. 2b, left) and two regions (Region \#1: Chr 3: 1,946,000 - 5,956,000; Region \#2: Chr 
$1113: 11,883,000-14,502,000)$ in the chromosome $3 p 25.1$ with a genetic length of 6.7 and $3 \mathrm{cM}$, each

112 displaying a significant LOD score greater than 3.0, respectively, in SB162 (Fig. 2b, right and

113 Extended Data Fig. 2a inset). Given the failure to show any link with the previously reported

114 ANSD genes $\left(\right.$ OTOF (NM_194248, 2p23.3) ${ }^{13}$, DIAPH3 (NM_001042517, 13q21.2) ${ }^{14}$,

115 SLC17A8 (NM_139319, 12q23.1) ${ }^{15}$, AIFM1 (NM_004208, Xq26.1) ${ }^{16}$, and OPA1 (NM_130837,

$1163 \mathrm{q} 29)^{17}$ ) and a new ANSD gene reported by us, $A T P 1 A 3\left(\mathrm{NM}_{-} 152296.4,19 \mathrm{q} 13.2\right)^{18}$, we

117 suspected an involvement of a novel gene.

118 Although the identification of a novel causative gene for ANSD from these two families was

119 done independently and blindly in China and South Korea, the linkage interval shared by the two

120 families narrowed down to $\mathrm{Chr} 3: 13,165,401-14,502,000$. Exome sequencing was first

121 performed from two affected subjects (\#36 and \#28) and one unaffected subject (\#22), yielding

122 five candidate variants co-segregating with the phenotype (XIRP1, CTBP2, CHODL, TMEM43

123 and $V I L L$ ) (Supplementary Table 1). Next, we performed detailed Sanger sequencing of these

124 variants from 10 additional subjects in HN66, leaving only one variant (p.R372X from

125 TMEM43) which co-segregated with the ANSD phenotype (Fig. 2c,d). This variant fell within

126 the linkage the linkage interval of HN66 (chr3: 13,165,401-22,769,511). For SB162, we

127 employed two diagnostic pipelines in a parallel fashion (Fig. 2a). First, we performed targeted

128 exome-sequencing of the two regions obtained from the linkage analysis among four subjects

129 (\#284, \#289, \#290, \#291), leading to identification of two nonsynonymous variants (TMEM43-

130 p.R372X and FBLN2-p.D851H) in Region \#2 that co-segregated with the ANSD phenotype

131 (Table 1, top). Of the two variants, we excluded FBLN2-p.D851H, based on its high occurrence

$132(0.4 \%)$ among the healthy Korean population in two independent databases, KRGDB ( $\mathrm{n}=1722)$

133 and in-house Samsung Genome Institute control subjects database $(n=400)$, leaving p.R372X 
134 from TMEM43 (NM_024334) as the only candidate. No convincing copy number variations,

135 such as a large genomic deletion and duplication, were detected within the locus (Extended

136 Data Fig. 2b). To exclude non-coding causative variants, we also performed whole genome

137 sequencing of the linkage interval from eight subjects (\#284, \#289, \#290, \#304, \#307, \#309,

138 \#324, \#332). The filtering step left us one nonsynonymous variant (TMEM43-p.R372X) and

139 eighteen non-coding region variants that co-segregated with the deaf phenotype within the

140 linkage interval (Supplementary Table 2). We excluded all of the eighteen variants, based on

141 their high occurrence $(>0.4 \%)$ among the two aforementioned Korean databases from normal

142 controls and detection of these variants among unaffected subjects or normal controls $(\mathrm{n}=65)$ by

143 Sanger sequencing, again leaving p.R372X from TMEM43 (NM_024334) as the only candidate

144 (Supplementary Table 2). Separately from the first pipeline and independently of linkage

145 analysis, we performed exome sequencing from the same four subjects (\#284, \#289, \#290, \#291),

146 identifying 20 possible candidate variants through an extensive filtering process in SB162 (Table

147 1, bottom). We performed detailed Sanger sequencing of these variants from 14 additional

148 subjects in the SB162, again leaving only one variant (p.R372X from TMEM43) as a candidate

149 (Fig. 2c,d and Extended Data Fig. 1b).

Along with the mutation spectrum of TMEM43 associated with the heart diseases reported in

151 HGMD database (Fig. 2e, top, black letter), the arginine at position 372, as well as amino acids

152 in the fourth transmembrane domain (TM) and C-terminal of TMEM43, is highly conserved

153 across organisms (Fig. 2e, bottom). A 3D modeling of human TMEM43 showed that p.R372X,

154 which was expected to introduce a premature stop codon, caused a truncation of the last 29

155 amino acids of the TMEM43 protein. (Fig. 2e, inset). This variant was predicted to be

156 pathogenic, according to the guideline for the interpretation of classifying pathogenic variants 
157 (PS3, PM2, PP1_Strong and PS4_Supporting) ${ }^{19,20}$ and high CADD score of 45

158 (https://cadd.gs.washington.edu/info) ${ }^{21}$. Previously, p.S358L in TMEM43 has been shown to

159

160

161

162

163

164

165

166

167

168

169

170

171

172

173

174

175

176

177

178

179

cause familial arrhythmogenic right ventricular cardiomyopathy (ARVC) ${ }^{22,23}$. However, our ANSD subject of HN66 and SB162 displayed symptoms of neither arrhythmia nor any other heart abnormalities. Conversely, hearing loss has not been reported in TMEM43-p.S358L ARVC patients ${ }^{22,23}$. This suggests that p.S358L and p.R372X of TMEM43 exert a completely different pathophysiological mechanism, leading to pleiotropy of this gene.

\section{TMEM43 is expressed in GLSs}

The cochlear expression of TMEM43 was firstly confirmed by RT-PCR (Fig. 3a). TMEM43 was expressed throughout the mouse body including cochlea, heart, eye and brain (Fig. 3a). To specify TMEM43 expression in the cochlea tissue, we performed immunostaining using antiTMEM43 antibody with epitope targeting p100-p200 (Ab $\left.\mathrm{A}_{1-1}\right)$. The expression of TMEM43 protein was evaluated at various time points along the postnatal development of hearing in the mouse cochlea (Fig. 3b-d). The previous studies have reported that TMEM43 is localized predominantly to the inner nuclear membrane in multiple non-cardiac cell types and also shows some expression outside the nucleus, including the endoplasmic reticulum ${ }^{23,24}$. In the case of $e x$ vivo cochlea, TMEM43 was mainly expressed in the organ of Corti, along the entire cochlear length, at postnatal day 4 (P4) through P20 (Fig. 3b-e). At P20, the expression became more restricted to the subset of the inner supporting cells (a subtype of GLSs) of the cochlea, which was the apical membrane of the inner border cells and the cell junctions of the inner sulcus cells (Fig. 3d). Throughout the early developmental period, up to P20, the TMEM43 expression was found exclusively in GLSs, but not in the hair cells, at both protein and mRNA levels. (Fig. 3b-i 
and Supplementary Video 1, 2). Adult mice at 1, 2, and 4 months still maintained this restricted expression in GLSs (Extended Data Fig. 3a). This glia-specific TMEM43 expression was recapitulated in adult primates corresponding to age 40's in humans, showing exclusive expression in the junctional area between the inner supporting and IHCs, without any overlap with calretinin staining (Extended Data Fig. 3b). TMEM43 expression in cochlear glial cell was confirmed again by co-expression with glia specific GFAP signal (Extended Data Fig. 3c). The specificity of the antibody was confirmed via a significant reduction of immunoreactivity from the cultured mouse organ of Corti when infected with lentivirus carrying Tmem43-shRNA (Extended Data Fig. 4a,b). These results strongly suggest that TMEM43 may play a critical role in cochlea GLSs.

\section{Localization of TMEM43 in the plasma membrane and loss of protein stability in TMEM43}

\section{p.R372X}

Based on hydrophobicity analysis, the secondary structure of TMEM43 was predicted to have four transmembrane domains and one intramembrane domain with its $\mathrm{N}$ - and $\mathrm{C}$-terminal domains located at extracellular space ${ }^{22}$ (Extended Data Fig. 5a). Bioinformatics revealed that TMEM43 was phylogenetically close among many species (Extended Data Fig. 5b) and the four transmembrane domains were highly conserved among different species (Extended Data Fig. 5c). We verified the predicted TMEM43 topology through immunocytochemistry with or without cell permeabilization. FLAG-tagged TMEM43 was transfected to HEK293T and was double stained with anti-FLAG and anti-TMEM43 with epitope targeting p203-p308 (Ab $\left.\mathrm{b}_{1-2}\right)$, at intracellular loop1 (Fig. 4a). We found that TMEM43 immunoreactivity was positive only with cell permeabilization, indicating that the loop1 of TMEM43 resides at the intracellular space 
(Fig. 4b). In addition, a positive FLAG signal without cell permeabilization was detected (Fig. 4b), indicative of membrane expression of TMEM43 and confirming the topology. To verify the cell-surface localization of TMEM43, we performed cell surface biotinylation assay. We found that TMEM43 wildtype (WT) protein trafficked to the plasma membrane, whereas TMEM43p.R372X showed almost complete disappearance of the biotinylated protein along with a significant reduction in total protein (Fig. 4c). Analysis of protein stability by the cycloheximide chase $(\mathrm{CHX})$ assay showed that this reduction was due to a decreased protein stability of TMEM43-p.R372X (Fig. 4d). Together, these results explain the etiology of TMEM43-p.R372X by reduction of protein expression at plasma membrane due to decreased protein stability.

\section{Generation of p.R372X knock-in mice displaying morphological change in GLSs with}

\section{progressive hearing loss}

To examine the pathogenic effect of mutant TMEM43 on the in vivo function of cochlea, a knock-in (KI) mouse harboring the human variant, p.R372X of TMEM43 in the corresponding mouse Tmem43 sequence (C57BL/6J;129S-Tmem43 ${ }^{\text {tmlCby }}$ ) was successfully established (Fig. 5a). The mutant TMEM43 protein was present in the GLSs till the adult period, as shown by a positive immunostaining from Tmem $43^{K I}$ mouse models (Fig. 5b, Extended Data Fig. 3d). Scanning electron microscopic (SEM) examinations showed that the apical surface area of GLSs, specifically the inner border cells of the organ of Corti from Tmem $43^{+/ K I}$ became significantly narrower than that of Tmem $43^{+/+}$from 7 months of age corresponding to mid-thirties in humans. This reduced size of GLSs became more prominent at 13 months of age in Tmem $43^{+/ K I}$ (Fig. 5c,d). In contrast, the shape and the arrangement of hair cell stereocilia as well as the number of synaptic ribbons were not different between the two genotypes (Extended Data Fig. 6a,b). 
Moreover, there was no sign of severe degeneration of OHCs up to 13 months in $T m e m 43^{+/ K I}$ mice compared with the littermate controls (Tmem $43^{+/+}$mice) (Extended Data Fig. 6c). These results indicate that p.R372X KI mice display morphological dysfunction specifically in GLSs. Finally, we observed a tendency of elevation of ABR thresholds starting at frequencies of 16 and $32 \mathrm{kHz}$ from both $T m e m 43^{+/ K I}$ and $T m e m 43^{K I / K I}$ mice compared with littermate Tmem $43^{+/+}$ control mice at 6 months of age which matches the onset time of hearing loss in human patients (Fig. 5e,f). Significantly reduced wave I amplitude from $T m e m 43^{+/ K I}$ and $T m e m 43^{K I K I}$ compared with that from $\mathrm{Tmem} 43^{+/+}$was noted, even at $8 \mathrm{kHz}$ at 6 months of age (Fig. $\mathbf{5 g}$ ), but not at 8 $\mathrm{kHz}$ at 3 months of age (Fig. 5h). The DPOAE responses representing the function of outer hair cells in both $T m e m 43^{+/ K I}$ and $T m e m 43^{K I / K I}$ mice were significantly higher than the noise level at the frequencies from 7.2 to $9.7 \mathrm{kHz}$, suggesting preservation of outer hair cell function. There was no significant difference in the sound levels of DPOAE among Tmem $43^{+/+}, \operatorname{Tmem} 43^{+/ K I}$, and Tmem $43^{K I / K I}$ mice (ANOVA, $\mathrm{p}>0.05$ ) at whole $\mathrm{kHz}$ (Extended Data Fig. 6d). These features of KI mice reflect the characteristics of ANSD $^{25}$, also indicating a similar pathogenic effect of the human TMEM43 variant on mice. In contrast, the electrocardiography of the Tmem $43^{+/ K I}$ mice did not show any sign of ARVC (Extended Data Fig. 7a), again confirming a profoundly distinct pathogenic effect of p.R372X compared to p.S358L.

\section{TMEM43 is an essential component of connexin channels in cochlear GLSs}

TMEM43 has been previously shown to be critical for gap junction channel function in cardiac muscles ${ }^{23}$, raising a possibility that pathogenic effect of TMEM43-p.R372X involves gap junction channels in cochlea such as $\mathrm{Cx} 26$ or $\mathrm{Cx} 30^{26}$. Therefore, we examined the localization of TMEM43 with connexin channels. Immunostaining data showed that TMEM43 
was expressed in GLSs along with Cx26 and Cx30 (Fig. 6a). In addition, the cochlea of Tmem $43^{+/+}$, Tmem $43^{+/ K I}$ and Tmem $43^{K I / K I}$ all showed similar Cx26 expression level (Fig. 6b). To directly confirm the physical interaction of TMEM43 with these connexin channels, we next performed co-immunoprecipitation assay. Both TMEM43 WT and TMEM43-p.R372X protein were immunoprecipitated with either Cx26 or Cx30 protein (Fig. 6c). These results together indicate that TMEM43 interact with connexin channels.

It has been reported that $\mathrm{K}^{+}$channel expression is low in GLSs so most of their resting membrane conductance is mediated by gap junctions, and gap junction blockers or isolation of GLSs increase their membrane resistance dramatically ${ }^{27}$, implying that gap junction channels mediate passive conductance current in GLSs. To test a possible involvement of TMEM43 in connexin channel function, we performed whole-cell patch clamp in GLSs from acutely dissected cochlea tissue by whole-cell patch clamp. Indeed, the large passive current with a linear current-voltage (I-V) relationship was recorded from GLSs, which was abolished when gap junction channel blocker carbenoxolone was treated (Fig. 6d,e). As the GLS gap junction networks are known to take part in recirculation of cochlear $\mathrm{K}^{+}$ions ${ }^{1}$, we treated a broadspectrum non-selective cation channel blocker $\mathrm{GdCl}_{3}{ }^{28}$. Treatment of $\mathrm{GdCl}_{3}(30 \mu \mathrm{M})$ also abolished the passive current of GLSs (Fig. 6f,g). We next examined if pH change can gate the passive conductance of GLS as some connexin channels are reported to be $\mathrm{pH}$ sensitive ${ }^{1}$. When extracellular $\mathrm{pH}$ was lowered to 6 , the passive conductance current was completely abolished

(Fig. 6h,i). These results indicate that GLSs display the large passive conductance current which is cationic and $\mathrm{pH}$ sensitive. The similar elimination of the passive conductance current was observed when cultured cochlea tissue was infected with a virus carrying Tmem 43 shRNA (Fig. $\mathbf{6 j}, \mathbf{k})$. We subsequently measured the passive conductance current from $T m e m 43^{K I}$ mouse. The 
272 passive conductance current from GLSs of $T m e m 43^{+/+}$was significantly reduced by $89 \%$ in

273 Tmem $43^{K I / K I}$ mouse (Fig. 6l,m), indicating that TMEM43 contributes majorly to the passive

274 conductance of the GLSs. The heterozygote mouse, Tmem $43^{+/ K I}$, which mimics the counterpart

275 human ANSD subjects, showed a significant reduction of $63 \%$ in the passive conductance

276 current in GLSs (Fig. 6l,m), confirming the significant pathogenic effect of the TMEM43 variant

277 on the function of GLSs. To rescue the impaired TMEM43-p.R372X-mediated current, we

278 generated a shRNA specifically targeting the KI sequence of Tmem $43^{K I}$ mouse. This Tmem $43 \mathrm{KI}$

279 shRNA targets only KI allele but not WT allele (Extended Data Fig. 4c,d, Fig. 6n,q). When

280 Tmem $43^{+/ K I}$ heterozygote cochlea culture was infected with the virus carrying Tmem43 KI

281 shRNA, the reduced passive conductance current was fully rescued to the level comparable to

$282 T$ Tmem $43^{+/+}$(Fig. 6o,q) but not in Tmem $43^{K I / K I}$ homozygote cochlea culture (Fig. 6p,q). These

283 results clearly explains the autosomal dominant effect of TMEM43-p.R372X and emphasize the

284 role of TMEM43 as an essential component of connexin channels in mediating the passive

285 conductance current in GLSs.

\section{Cochlear implant restores the impaired speech discrimination in the ANSD subjects}

291 severe to profound sensorineural hearing loss, as a result of significant damages to OHCs or

292 IHCs, would require a cochlea implant (CI). A subset of sensorineural hearing loss mainly

293 affecting more central structures, such as spiral ganglion neuron or cochlear nerve, or central

294 hearing loss originating from the brainstem or brain, may benefit from brainstem implant, but not 
295 from either hearing aids or CI. Because the main damage in Tmem $43^{+K I}$ mouse was restricted to 296 GLSs, but not to the hair cells or spiral ganglion neurons at minimum $6 \sim 7$ months of age, we 297 could predict that subjects from SB162 could benefit from CI. Consequently, CI was performed 298 on \#284 and \#304, even though the PTA of \#284 did not meet a conventional criterion of CI 299 (PTA equal to or exceeding $70 \mathrm{~dB}^{29}$ ). Postoperatively, the open-set speech understanding of $300 \quad \# 284$ with a deaf duration of 15 years more rapidly restored than that of control adult cochlear 301 implantees $(n=39)$, as measured by speech evaluation test tool ${ }^{30,31}$ (Fig. 7a,b). However, the 302 restoration of speech discrimination ability in \#304 with a longer deaf duration (about 25 years) 303 was not as rapid as that in \#284 (Fig. 7c). To interest, the responsiveness of cochlear nerve to 304 stimuli of \#304, measured by intracochlear electrically evoked ABR (e-ABR), was restored 305 immediately after cochlear implantation (Fig. 7d), which sharply contrasted with other ANSD 306 subject with a postsynaptic etiology (Fig. 7e). These results suggest that limited improvement of 307 speech discrimination in \#304 is presumably due to cortical damage related with longer deaf 308 duration, but not due to cochlear implant-related issues. Based on our results, early cochlear 309 implantation may be recommended despite substantial residual hearing, in the case of TMEM43310 p.R372X among adult onset, progressive ANSD subjects.

\section{Discussion}

313 In this study, we have identified a novel deafness locus, Chr 3: 13,165,401 -14,502,000 (3p25.1)

314 and a new deafness gene, TMEM43, which, if altered, can cause ANSD in adults. The p.R372X

315 variant of TMEM43 is considered clearly pathogenic and causative of ANSD of HN66 and

316 SB162, based on the guideline for the interpretation of classifying pathogenic variants (PS3,

317 PM2, PP1_Strong and PS4_Supporting) ${ }^{19,20}$. Detection of this variant from two unrelated 
318 families (each from China and South Korea) sharing rare and characteristic ANSD phenotype

319 significantly increases causality between this variant and ANSD. Unbiased exome sequencing

320 screening was performed both for HN66 and SB162 in a parallel fashion with linkage analysis to

321 minimize errors from spurious linkage. Significant overlap of linkage interval between HN66

322 and SB162 in Chr 3: 13,165,401 -14,502,000 made it least likely for our linkage data to be

323 reflection of spurious linkage. Further, we have identified and characterized TMEM43 as an

324 essential component of connexin channels contributing to the passive conductance current in

325 GLSs which is necessary for hearing and speech discrimination. Until now, only the pathological

326 functions of TMEM43 has been implicated in arrhythmia ${ }^{22,23}$ and tumor progression ${ }^{32}$. The

327 variant p.S358L of TMEM43 has been reported to cause ARVC by decreasing the expression and

328 localization of tight junctions, redistributing the gap junction proteins from the surface to the 329 cytoplasm, and decreasing the conduction velocity ${ }^{23}$.

330 Our study firstly demonstrates the presence of the $\mathrm{CBX}-, \mathrm{GdCl}_{3}$ - and low $\mathrm{pH}$-sensitive 331 passive conductance current in the GLSs of intact cochlea. The high expression of passive 332 conductance channels, which gives rise to very low membrane resistance and linear I-V 333 relationship, is known as the unique property that defines mature astrocytes in the brain ${ }^{33}$.

334 Therefore, our findings imply that GLSs display similar properties as mature astrocytes.

335 Consistently, GLSs express GFAP and GLAST ${ }^{34}$, which are common markers for mature 336 astrocytes. In the brain, passive conductance channels are thought to be involved in $\mathrm{K}^{+}$ion 337 homeostasis ${ }^{35}$ and volume regulation ${ }^{36,37}$. In the cochlea, the endocochlear potential of $+80 \mathrm{mV}$ 338 is generated by maintaining high $\mathrm{K}^{+}$concentration in the endolymph, and this potential is critical 339 for activation of hair cells ${ }^{38}$. The presence of passive conductance current implicates an 340 important role of GLSs in homeostasis of $\mathrm{K}^{+}$and volume regulation. We have observed a 
341 significantly smaller inner border cell size of Tmem $43^{K I}$ mice compared to control, with loss of

342 passive conductance current. We hypothesize that the smaller inner border cells have smaller

343 capacity for $\mathrm{K}^{+}$uptake. The loss of the passive conductance current indicates impaired recycling

344 of $\mathrm{K}^{+}$. Such breakdown of $\mathrm{K}^{+}$homeostasis would make hair cells difficult to depolarize, resulting

345 in a disruption of speech discrimination ability.

It is of interest that the onset time of pathology and hearing loss in heterozygous Tmem $43^{K I}$ mouse (6 7 months of age which equals mid-thirties in human) precisely matches the onset time of a full extent of ANSD (mid-thirties) in our affected subjects. This raises a fundamental question of why canonical ANSD does not occur in young age. The passive conductance current recordings from the cochlea of $\mathrm{P} 5-7$ pups revealed a $63 \%$ reduction in passive conductance

351 current in heterozygous Tmem $43^{K I}$ mice. We also showed that lowering the $\mathrm{pH}$ to 6 reduced the passive current of GLS. The late-onset hearing loss could be explained by a reduction of cochlear $\mathrm{pH}$ in aging mouse and human, similar to what happens in aging brain ${ }^{39}$. The normal hearing in heterozygote Tmem $43^{K I}$ mouse and the ANSD subjects at young age could be owing to the remaining passive conductance current that might be enough to maintain homeostatic functions of GLSs. In contrast, the hearing loss in the aging Tmem $43^{K I}$ mice and ANSD subjects can be explained with further reduction of the remaining current due to the low $\mathrm{pH}$-sensitivity,

358 impairing the $\mathrm{K}^{+}$homeostatic in GLSs. These exciting possibilities await future investigations.

360 patients. More importantly, our study suggests that if a causative gene of ANSD is expressed 361 mainly at GLSs, it could also be used as a favorable prognostic marker for CI. Although CI 362 successfully rehabilitates severe to profound sensorineural hearing loss in many cases, especially 363 adult ANSD patients usually hesitate to receive the surgery due to uncertainty of surgery 
364 outcome regarding speech discrimination, side-effects including loss of residual hearing, and

365 high cost. Given this, an ANSD-causative variant of GLS-specific gene such as p.R372X of

366 TMEM43 could serve as a potential indicator of positive CI surgery outcome if the surgery is

367 timely done. In this sense, our study also proposes the optimal time of CI surgery: \#284 who

368 received CI at the age 43 recovered his sentence understanding ability to $100 \%$ (Fig.7a), while

369 \#304 who received CI at the age 53 recovered her ability to $22.2 \%$ one year after surgery (Fig.

370 7c,d). Relatively slow functional recovery from \#304 could be attributed to the cortical damage

371 due to longer deaf duration. Thus, we may have to recommend the ANSD patients carrying

372 TMEM43-p.R372X to receive a CI surgery as soon as they start to experience significantly

373 diminished speech discrimination even though they retain significant residual sound detection

374 ability. Our study provides an important step towards identifying more TMEM43-related ANSD

375 patients, enabling further study with larger number of subjects carrying this allele.

376 In conclusion, we have characterized TMEM43 as an essential component of connexin

377 channels and have delineated that an alteration of this protein causes functional and

378 morphological abnormalities in GLSs, resulting in the failure to maintain speech discrimination

379 in aged human. Through these mechanistic insights, we have elucidated a link between

380 abnormality in cochlear GLSs and impaired human speech discrimination. We further provide a

381 model platform in which the personalized timing and mode of auditory rehabilitation can be

382 determined, highlighting the importance of a precision medicine-based approach. 


\section{Methods}

Family data. Clinical examination was performed on a total of 18 subjects: 6 male and 12 female subjects whose age ranged between 22 and 84 years from family SB162. Five affected subjects with progressive "ANSD" and 11 unaffected subjects in a 5 generation Chinese Han HN66 family participated in the present study. Informed consent was obtained from all participants in this study. Pure-tone audiometry was carried out to evaluate the air and bone conduction thresholds. The binaural mean pure-tone average of thresholds for air conduction (in dB SPL) was calculated for audiometric frequencies of $0.25,1,2$, and $4 \mathrm{kHz}$ (PTA $0.25,1,2$, 4kHz). The speech discrimination score was measured whenever possible. Hearing impairment was additionally assessed by the means of ABRs and DPOAEs. The ABR threshold is a minimal amplitude of sound that can elicit the wave V response. Otoscopic examination and tympanometry with acoustic reflex testing were performed systematically to rule out any conductive hearing impairment. To evaluate the function of outer hair cells, sound pressure of DPOAE and surrounding noise were evaluated using a SmartEP (IHS, Miami, FL). An Etymotic

$39810 \mathrm{~B}+$ microphone was inserted to measure sound pressure in the external ear canal, and pure tone from 3.6 to $14.4 \mathrm{kHz}$ was presented using Etymotic ER2 stimulator. Frequencies were 400 acquired with an F2-F1 ratio of 1.22. Stimulation levels ranged from 65 to $25 \mathrm{~dB}$ SPL at 10-dB 401 interval. This study was approved by the institutional review board of Seoul National University 402 Bundang Hospital (IRB-B-1007-105-402) and the ethics committee of Xiangya Hospital of 403 Central South University (ID: 201603518). 
Linkage analysis. The Infinium Global Screening Array panel (Illumina, San Diego, CA), consisting of 660 thousands of SNPs, was used to conduct a whole genome linkage scan with 18 subjects (10 affected and 8 unaffected) in SB182 and 9 subjects (5 affected and 4 unaffected) in HN66. The genomic DNAs were extracted from the peripheral blood. Multipoint $\log _{10}$ Odds (LOD) scores were computed using MERLIN (version 1.1.2) and was given for all genotyped positions. Analyses were performed assuming the dominant traits based on family history. The disease allele frequency was set to 0.0001 , and the penetrance for homozygous normal, heterozygous, and homozygous affected were set to $0.0001,1.0$, and 1.0 respectively. Family members with unclear status were coded as unknown. Because Merlin allows a maximum of 24 bits for a family by default, the family was split into sub-families having common ancestors in SB162.

Targeted exome sequencing and exome sequencing. Targeted exome sequencing of the critical region 3p25.1 from the linkage analysis for four subjects (SB162-\#284, \#289, \#290, \#291) was performed and the candidate variants were listed (Table 1). The variants $<1 \%$ minor allele frequency in ESP6500 and 1000G were initially identified. Only inheritance pattern-matched variants were remained, and the variants in dbSNP, but not in flagged dbSNP were filtered out. We used our in-house database having KRGDB_1722 individuals to remove Korean-specific common variants. In addition to single nucleotide variation (SNV), possible presence of copy number variations $(\mathrm{CNV})$ was also identified using Excavator ${ }^{8}$. A genome level $\log _{2}$ ratio for chromosome 3 was investigated. The subjects proband was screened for hotspot variants causing Hearing Loss in Korean and Chinese population via hot spot screening, which include GJB2, SLC26A4 and mitochondrial 12S rRNA. In parallel, being blind to the linkage analysis results, 
we also performed exome sequencing on the four subjects (SB162-\#284, \#289, \#290, \#291) in SB162 and 2 affected subjects (\#36 and \#28) and 1 unaffected subject (\#22). Bioinformatics analyses were performed as previously described ${ }^{8}$. Thereafter, the final candidate variants in these families were verified using Sanger sequencing. The variants in non-coding regions as well as synonymous variants in coding regions were filtered out. Variants with a minor allele frequency of less than $1 \%$ were selected based on the Exome Sequencing Project 6500 (ESP6500), 1000 Genome Project (1000G), Exome Aggregation Consortium (ExAC), and our in-house database containing the exomes of 81 Korean individuals. Based on the autosomal dominant inheritance pattern, single heterozygous variants with sufficient read depths $(>10 \mathrm{X})$ and genotype quality $(>20)$ were selected when they were commonly found in all the affected siblings. Further, variants of dbSNP ID with no clinical significance were filtered out. Finally, 20 possible candidate variants in SB162 and 5 candidates in HN66 that co-segregated with ANSD phenotype remained (Table 1 and Supplementary Table 1). To ascertain the candidate pathogenic variants, ES was performed on two affected subjects (\#36 and \#28) and one unaffected subject (\#22) and the resulting sequences were processed using the pipeline that we have previously described ${ }^{40}$. After initial quality control, variants were firstly filtered using the deafness-associated gene list (DAGL) to search for variants in known deafness genes. Then, the remaining variants were filtered according to the criteria as follows: (1) locating at the coding exons and intron-exon adjacent regions; (2) MAF $<0.1 \%$ in multiple databases including the Exome Aggregation Consortium (EXaC) (http://exac.broadinstitute.org/), the 1000 Genome Project (http://browser.1000genomes.org), gnomAD (http://gnomad.broadinstitute.org/) and the ATCG (Annoroad Typical Chinese Genomes) database; (3) heterozygous variants in the form of non-synonymous, non-sense, splice sites, Indels, codon mutations; (4) predicted pathogenicity 
451 for missense mutations using SIFT (http://sift.jcvi.org), MutationTaster

452 (http://mutationassessor.org), and Polyphen2 (http://genetics.bwh.harvard.edu/pph2).

453 Candidate pathogenic variants were further conformed by mapping the filtered variants obtained

454 from WES to the linkage regions.

455

Whole Genome Sequencing. Whole genome sequencing was performed on 8 DNA samples (alignment to the hg19 reference genome, deduplication, local de novo assembly, and variant calling) was performed using BWA, picard, and GATK). Variants firstly were restricted to those in specified regions (chr3:11,883,000-chr3:14,502,000, based on the result of linkage analysis).

461 We then filtered out the variants that were not matched to inheritance patterns, and that were in 462 Korean reference database (KRGDB, KOVA and in-house Samsung Genome Institute control 463 subjects database) and the $1000 \mathrm{G}$ project.

Evaluation of speech discrimination. Speech performance was evaluated preoperatively, and 3, 
Animals and housing. Tmem43-R372X Knock-in (C57BL/6J;129S- Tmem $\left.43^{\text {tm ICby }}\right)$ mice, 129Sv/Ev mice and crab-eating macaque were used. All animals were kept on a 12 hours lightdark cycle in a specific-pathogen-free facility with controlled temperature and humidity and had free access to food and water. All experimental procedures were conducted according to protocols approved by the directives of the Institutional Animal Care and Use Committee of Seoul National University Bundang Hospital (Seongnam, Republic of Korea) and the Institutional Animal Care and Use Committee of IBS (Daejeon, Republic of Korea). As the sequence difference between $\operatorname{Tmem} 43^{+/+}$and $\operatorname{Tmem} 43^{K I / K I}$ was too small to be detected by conventional PCR method, mouse cDNA was amplified with primers (F: 5gtttatgggcctcaacctcatg-3, R: 5- caggcttcactccagcttttgg-3) and then enzyme digested with BsiEI (Thermofisher \#FD0894). Genotyping result using this method is shown in Fig. 5a. The expected band size for WT is $90 \mathrm{bp}$ and $123 \mathrm{bp}$, and for $\mathrm{KI} / \mathrm{KI}$ is $213 \mathrm{bp}$.

\section{Construction of Tmem43-R372X knock-in mice model. The Tmem43-R372X Knock-in}

(C57BL/6J;129S- Tmem $43^{\text {tmlCby }}$ ) mouse model was generated using Clustered Regularly Interspaced Short Palindromic Repeats (CRISPR) technology. In such a model, Arginine (R) was replaced with the STOP codon at position 372 of the TMEM43 protein (gene ID: NM_028766).

The criteria used for gRNA selection are the distance to the modification site and the off-target profile. Based on these guidelines, two gRNA candidates (Tmem43 gRNA1:5'-

\section{TTAGAGCAGCCCACAGCGGTCGG-3'; Tmem43 gRNA2: 5'-}

CCGATTAGAGCAGCCCACAGCGG-3'), located in proximity to the mutation site, were selected and evaluated. To determine gRNA activity, a SURVEYOR assay was performed, using SURVEYOR mutation detection kit (IDT), according to the manufacturer's instructions. Based 
on the validated gRNAs, a single stranded oligodeoxynucleotide donor (ssODN) was designed and synthesized. A $160 \mathrm{bp}$ donor contains two homology arms flanking introduced $(\mathrm{C} \rightarrow \mathrm{T})$ mutation in exon 12 corresponding to 1114 position in the Tmem43 gene. Moreover, to protect the repaired genome from being re-targeted by Cas9 complex, additional mutations were introduced corresponding to a second stop codon. The $62 \mathrm{C} 57 \mathrm{BL} / 6 \mathrm{~J}$ embryos were injected through a cytoplasmic route with a CRISPR cocktail containing ssODN donor, gRNA transcripts Tmem43 and Cas9 mRNA. Forty-nine out of 62 embryos passed the quality screening and were implanted into two surrogate CD1 mice. From this round of microinjection, all females became pregnant, and gave birth to nine pups F0. To confirm the germline transmission of the transgene, female founder F0 was mated with the wild type C57BL/6J male and subjected to F1 breeding to confirm germline transmission of the transgene. All animals were analyzed by PCR and sequencing for the presence of correct point mutation at the target site. For genotyping, genomic DNA was extracted from 1- to 2-mm-long tail tips using the DNeasy ${ }^{\circledR}$ Blood \& Tissue kit (Qiagen, Hilden, Germany). Genomic DNA ( $5 \mu \mathrm{l})$ was analyzed by PCR in a final volume of 50 $\mu \mathrm{l}$ in the presence of $25 \mathrm{mM} \mathrm{MgCl} 2$ and $2 \mathrm{mM}$ dNTPs, at $10 \mathrm{pM}$ of each primer, and $0.02 \mathrm{U}$ of TOYOBO KOD Hot Start DNA polymerase (Invitrogen/Life Technologies, Billerica, Massachusetts, USA) with primers: Forward (5'-CCACAGTGGACTGGTTTCCT-3') and Reverse (5'-GGCTTCACTCCAGCTTTTTG-3') detecting the presence of the knock-in allele (213 base pairs). After a denaturing step at $95^{\circ} \mathrm{C}$ for 2 minutes, 35 cycles of PCR were performed, each consisting of a denaturing step at $95^{\circ} \mathrm{C}$ for 20 seconds, followed by an annealing phase at $59^{\circ} \mathrm{C}$ for 10 seconds and an elongation step at $72^{\circ} \mathrm{C}$ for 10 seconds. PCR was finished by a 10 -minute extension step at $72^{\circ} \mathrm{C}$. Amplified products were confirmed by Sanger sequencing (Macrogen Inc., Seoul, KOR). 
Plasmids. TMEM43 (Myc-DDK-tagged)-Human transmembrane protein 43 (TMEM43)

(GenBank accession no. NM_024334.2) was purchased from OriGene (RC200998) and cloned into CMV-MCS-IRES2-EGFP vector using BglII/XmaI sites. hTMEM43-p.R372X mutation and p.401X mutation (to put stop codon before FLAG) were obtained by performing oligonucleotidedirected mutagenesis using the EZchange site-directed mutagenesis kit (EZ004S, Enzynomics). mTMEM43 (GenBank accession no. NM_028766.2) was obtained by using a RT-PCR based gateway cloning method (Invitrogen) and cloned into CMV-EGFP-C1 vector using BglII/SalI sites. GJB2 (NM_004004) Human Tagged ORF Clone was purchased from OriGene (RC202092) and Cx30-msfGFP was purchased from Addgene (69019). (HEK) 293T cells were purchased from ATCC (CRL-3216). The cell line has been tested for mycoplasma contamination. HEK293T cell was cultured in DMEM (10-013, Corning) supplemented with 10\% heat-inactivated fetal bovine serum (10082-147, Gibco) and 10,000 units/ml penicillin-streptomycin $\left(15140-122\right.$, Gibco) at $37^{\circ} \mathrm{C}$ in a humidified atmosphere of $95 \%$ air and 5\% $\mathrm{CO}_{2}$. Transfection of expression vectors was performed with Effectene Transfection

536 Reagent (Effectene, 301425, Qiagen), according to the manufacturer's protocol. One day prior to 537 performing the experiments, HEK293T cells were transfected with each DNA $1.5 \mu \mathrm{g}$ per $60 \mathrm{~mm}$ 538 dish. Ratio of DNA to Effectene Reagent is 1:10.

RT-PCR. The $129 \mathrm{~Sv} / \mathrm{Ev}$ mice were deeply anesthetized with pentobarbital sodium $(50 \mathrm{mg} / \mathrm{kg})$.

541 Total RNA was extracted from their whole cochlea (P1), heart (P120), eye (P42), brain (P28), 
542 kidney (P28), and liver (P28) to analyze the expression of the target mRNAs using TRIZOL ${ }^{\circledR}$

543 (Gibco BRL, Gaithersburg, MD, USA) and a column from an RNeasy Mini Kit (Qiagen,

544 Valencia, USA), performed in accordance with the manufacturer's instructions. The RNA

545 obtained was used to synthesize cDNA with oligo (dT) primers, one-twentieth of which was used

546 for one PCR reaction. The DNA amplification was performed in a final volume of $30 \mu \mathrm{l}$. PCR

547 cycling conditions were $95^{\circ} \mathrm{C}$ for $2 \mathrm{~min}$, followed by 35 cycles of $95^{\circ} \mathrm{C}$ for $20 \mathrm{~s}, 55^{\circ} \mathrm{C}$ for $10 \mathrm{~s}$,

548 and $70^{\circ} \mathrm{C}$ for $10 \mathrm{~s}$, with a final step of $72^{\circ} \mathrm{C}$ for $5 \mathrm{~min}$. Amplified product $(15 \mu \mathrm{l})$ was separated

549 using electrophoresis in a $2 \%$ agarose gel and visualized with ethidium bromide. We analyzed

550 the transcripts of TMEM43 and GAPDH by primer pairs, as follows: the forward primer $\left(5^{\prime}-\right.$

551 CTTCCTGGAACGGCTGAG-3') and the reverse primer (5'-CACCAGCCTTCCTTCATTCT-

552 3') for Tmem43, and the forward primer (5'-ACCACAGTCCATGCCATCAC-3') and the

reverse primer (5'-CACCACCCTGTTGCTGTAGCC-3') for Gapdh.

Immunocytofluorescence on cochlear tissue. Primary antibodies used are rabbit anti-TMEM43 monoclonal (1:500, Ab184164, Abcam) (Ab $\left.{ }_{1-1}\right)$, rabbit anti- TMEM43 polyclonal (1:100, NBP184132, Novus) (Ab $1-2)$, mouse anti-mCherry monoclonal (1:500, ab125096, Abcam), mouse anticalretinin monoclonal (1:500, MAB1568, Millipore), goat anti-Na ${ }^{+}, \mathrm{K}^{+}$-ATPase- $\alpha 3$ polyclonal

559 (NKA, 1:250, SC-16052, Santa Cruz), and mouse anti-CtBP2 monoclonal (1:500, 612044, BD

560 Transduction Laboratories) antibodies. $A b_{1-1}$ was used in cultured cochlea and $A b_{1-2}$ was used in

561 freshly dissected cochlea. Secondary antibodies generated either in donkeys or goats (life

562 technologies) were used at 1:1000. Secondary antibodies used are donkey anti-mouse alexa fluor 563555 (A-31570, Thermo fischer scientific), donkey anti- rabbit alexa fluor 488 (A-21206, Thermo 564 fischer scientific), donkey anti-goat alexa fluor 633 (A-21082, Thermo fischer scientific), goat 
anti-rabbit alexa fluor 555 (A-21428, Thermo fischer scientific), goat anti-mouse alexa fluor 488 (A-11001, Thermo fischer scientific), alexa fluor 488 goat anti-rabbit $\operatorname{IgG}(\mathrm{H}+\mathrm{L})$ (A11008, Life technologies). No immunoreactivity was found when the primary antibodies were omitted. Mouse inner ears (C57BL/6) at various time points postnatally and the cochlea from three monkeys weighing $5 \mathrm{~kg}$ were fixed in ice cold $4 \%$ paraformaldehyde, for $1 \mathrm{~h}$. Cochlear turns were carefully excised and incubated in blocking/permeabilizing buffer (PBS with goat or donkey serum and $0.25 \%$ Triton $\mathrm{X}-100$ ). Then, the preparations were incubated overnight at $4^{\circ} \mathrm{C}$ with primary antibodies diluted in the blocking/permeabilizing buffer. After 3 washes, the cochlear turns were reacted with fluorescence labeled secondary antibodies diluted in blocking/permeabilizing buffer for $1 \mathrm{~h}$ at room temperature. Samples were then rinsed once with blocking/permeabilizing buffer and twice with PBS. Using Fluorsave reagent (Calbiochem, 345789), the tissues were mounted on glass slides and covered with coverslip. Specific immunolabeling was initially examined under epifluorescence microscope and high-resolution images were obtained using a confocal laser scanning microscope (LSM710, Zeiss).

Immunocytofluorescence on heterologous system. For immunocytochemistry, hTMEM43IRES2-EGFP was transfected into HEK293T cells one day prior to staining. Cells were fixed in 4\% paraformaldehyde for $30 \mathrm{~min}$ at room temperature and washed 3 times with PBS. The permeabilized group contained 3\% Triton X-100 in the blocking solution with 2\% goat serum and $2 \%$ donkey serum but the impermeabilized group excluded Triton $\mathrm{X}-100$ in the blocking solution. Cells were incubated with rabbit anti-TMEM43 polyclonal antibody (1:100, NBP184132, Novus) (Ab $\left.b_{1-2}\right)$ and mouse anti-FLAG (1:500, F1804, Sigma) for overnight. After washing, goat anti-Rabbit IgG Alexa Flour 647 (1:1000, A21429, Invitrogen) and donkey anti- 
Mouse Alexa 555 (1:1000, A31570, Molecular Probe) were added and incubated for 2 hours at room temperature. The cells were washed 3 times and mounted, and then observed under a Nikon A1 confocal microscope.

In situ hybridization. To make specific riboprobe for mRNA of Tmem43 and Spmx, we cloned partial cDNA fragments of Tmem43 and Spmx. Primers were as follows: Tmem43, forward: 5'TGTTCGTGGGGCTAATGACC-3; reverse: 5'-TAGTTGGTCACCTCGTTGCC-3', T7-reverse primer: 5'-TAATACGACTCACTATAGGGAGATCACAGTAACCACGTGAGCC-3'; Spmx, forward: 5 '-TTTCCACACGGTCAAGCCTTT-3; reverse: 5'TCTCCTCAAAACCACACTTCCC-3', T7-reverse primer: 5'TAATACGACTCACTATAGGGAGAGTCTTGGGCATTCAGGAGGTT-3'. The plasmid was linearized and used for in vitro transcription (Roche Dignostics, Indianapolis, IN, USA) to label RNA probes with digoxigenin-UTP. The $3^{\prime}$ and $5^{\prime}$ - DIG labeled miRCURY LNA miRNA

Detection probes (Qiagen, negative control Scramble-miR cat. \# YD00699004-BCG) were used for In situ hybridization. In situ hybridization was performed as previously described with some modifications. Frozen cochlea was sectioned at $20 \mu \mathrm{m}$ thickness on a cryostat. The sections were then fixed in $4 \%$ paraformaldehyde, washed with PBS, and acetylated for $10 \mathrm{~min}$. The sections were incubated with the hybridization buffer (50\% formamide, 4 X SSC, $0.1 \%$ CHAPS, $5 \mathrm{mM}$ EDTA, $0.1 \%$ Tween-20, $1.25 \times$ Denhartdt's, $125 \mu \mathrm{g} / \mathrm{ml}$ yeast tRNA, $50 \mu \mathrm{g} / \mathrm{ml}$ Heparin) and digoxigenin-labeled probes $(200 \mathrm{ng})$ for $18 \mathrm{~h}$ at $60{ }^{\circ} \mathrm{C}$. Non-specific hybridization was removed by washing in $2 \mathrm{X} \mathrm{SSC}$ for $10 \mathrm{~min}$ and in $0.1 \mathrm{X} \mathrm{SSC}$ at $50^{\circ} \mathrm{C}$ for $15 \mathrm{~min}$. For immunological detection of digoxigenin-labeled hybrids, the sections were incubated with anti-digoxigenin antibody conjugated with alkaline phosphatase (Roche Diagnostics) for $1 \mathrm{~h}$, and the colour 
611 reaction was carried out with NBT/BCIP (4-nitroblue tetrazolium chloride/bromo-4-chloro-3-

612 indolyl phosphate; Sigma). Sections were dehydrated and mounted with Vectamount (Vector

613 Laboratories, Burlingame, CA, USA).

614

615 Cochlear organotypic culture and shRNA treatment. Neonatal mouse (C57/BL6, P4)

616 cochlear turns were isolated in ice cold sterile Hanks' Balanced Salt Solution (HBSS). Cochlear

617 segments were attached on Cell-Tak (354240, Corning) coated coverslips and incubated

618 overnight in DMEM/F12 medium containing 1\% fetal bovine serum, $5 \mu \mathrm{g} / \mathrm{ml}$ ampicillin, B27 and

$619 \mathrm{~N} 2\left(37^{\circ} \mathrm{C}, 5 \% \mathrm{CO}_{2}\right.$ humidified incubator). Upon confirming stable attachment, the tissues were

620 treated with either scramble or shRNA diluted in culture medium (1:1000) for $48 \mathrm{~h}$. The medium

621 was then replaced with a fresh one. After additional $48 \mathrm{~h}$ of incubation, the tissues were

622 subjected to further examination.

623

624 Construction and packaging of shRNA-lentiviral vectors. For TMEM43 gene silencing,

625 candidate for both mouse and rat Tmem43 shRNA (GenBank accession no. NM_028766.2 and

626 NM_001007745.1) sequences were cloned into lentiviral pSicoR vector using XhoI/XbaI sites,

627 as previously described ${ }^{41}$. Knockdown efficiency was determined by a reduction in fluorescence

628 expression of CMV-EGFP- TMEM43 when overexpressed with Tmem43 shRNA candidate

629 containing pSicoR- Tmem43 shRNA-mCh vector. Validated shRNA candidate containing

630 pSicoR vector was packaged into high-titer lentivirus by KIST virus facility (Republic of Korea).

631 In brief, the lentiviral vectors were produced by co-transfecting each pSicoR vector with the

632 ViraPower lentiviral packaging mix (Invitrogen) in the 293FT packaging cell line. The

633 supernatant was collected and concentrated by ultracentrifugation. For efficient shRNA delivery, 
634 lentiviral vectors were always produced at subneutral $\mathrm{pH}(\leq \mathrm{pH} 7.0)$. The target regions of Tmem43 shRNA is 5'-GCTCTTGTCTGACCCAAATTA-3' and Tmem43 KI shRNA is 5'CTCTTCTACTGATGACTGTGG-3'. For control shRNA, scrambled sequence 5'TCGCATAGCGTATGCCGTT-3' was inserted in place of shRNA sequence.

Chemicals. Chemicals used in this study were all purchased from Sigma-Aldrich. $\mathrm{NaOH}$ ). All recordings were done with same HEPES buffer as external solution. Stria vascularis and tectorial membrane were carefully peeled off and the remaining cellular organization of the organ of Corti was left intact. Acutely dissected cochlea turn was used within 2 hours of dissection. Glia-like supporting cells that are located below inner hair cell layer were whole cell patch clamped and current traces were elicited by $1 \mathrm{sec}$ ramps ascending from $-100 \mathrm{mV}$ to

$649+100 \mathrm{mV}$ with $-60 \mathrm{mV}$ holding potential. Recording electrodes (7-11M $\Omega$ ) supplemented with 650 (mM): $126 \mathrm{KOH}, 126$ Gluconate, 5 HEPES, $0.5 \mathrm{MgCl}_{2}$ and 10 BAPTA (pH adjusted to 7.3 with $651 \mathrm{KOH}$ ) advanced through the tissue under positive pressure. Slice chamber was mounted on the 652 stage of an upright Hamamatsu digital camera viewed with an X60 water immersion objective 653 with infrared differential interference contrast optics using Imaging Workbench Software.

654 Electrical signals were digitized and sampled with Digidata 1320A and Multiclamp 700B 655 amplifier (Molecular Devices) using pCLAMP 10.2 software. Data were sampled at $10 \mathrm{kHz}$ and 
filtered at $1 \mathrm{kHz}$. Glass pipette were pulled from micropipette puller (P-97, Sutter Instrument) and all experiments were conducted at a room temperature of $20-22^{\circ} \mathrm{C}$.

Cell surface biotinylation, co-immunoprecipitation and western blot. For biotinylation, CMV-hTMEM43 WT-IRES2-EGFP or CMV-hTMEM43 R372X-IRES2-tdTomato vector were transfected into HEK293T cells 1 day prior to the experiment day. Transfected cells were washed three times with PBS and cell surface-expressed proteins were biotinylated in PBS containing Ez-link sulfo-NHS-LC-Biotin (21335, Thermo) for $30 \mathrm{~min}$. After biotinylation, cells were washed with quenching buffer (100mM glycine in PBS) to remove excess biotin and then washed three times with PBS. The cells were then lysed and incubated with high capacity NeutrAvidin-Agarose Resin (29204, Thermo). After three washes with lysis buffer, bound proteins are eluted by the SDS sample buffer and subjected to western blot analysis. Primary antibody used is: rabbit anti-TMEM43 polyclonal (1:100, NBP1-84132, Novus) ( $\left.\mathrm{Ab}_{1-2}\right)$. Secondary antibody used is: Donkey anti-rabbit HRP (NA9340, Amersham). For CHX assay, 40 ug/ml CHX was treated to TMEM43 WT and p.R372X expressing HEK293T cells and analyzed in 3 hours interval. Anti-FLAG M2 (1:2000, F1804, Sigma-Aldrich) was used for western blot. For co-immunoprecipitation, cell lysates were prepared in a buffer containing $50 \mathrm{mM}$ Tris- $\mathrm{HCl}$ (pH 7.5), $150 \mathrm{mM} \mathrm{NaCl}, 1 \% \mathrm{NP}-40,10 \mathrm{mM} \mathrm{NaF}$, and protease and phosphatase inhibitor cocktail. Equal amounts of precleared cell lysates were incubated with rabbit anti-FLAG (2368s, Cell Signaling) overnight. Protein A/G-Agarose beads (Thermo Fisher) were added to the mixtures and further incubated for $2 \mathrm{~h}$, followed by a wash with lysis buffer. Bound proteins were eluted from the beads with SDS-PAGE sample buffer and western blotting was performed with rabbit anti-TMEM43 $\left(\mathrm{Ab}_{1-2}\right)$. 
680 Scanning electron microscope (SEM) analysis. For SEM analysis, the organ of Corti were

681 performed using a method previously described ${ }^{42}$. Briefly, the cochleae were immediately

682 isolated from the euthanized Tmem $43^{+/+}$and $T m e m 43^{+/ K I}$ mice at 2,7 , and 13 months of age and

683 fixed in a solution of 2\% PFA dissolved in $0.1 \mathrm{M}$ sodium cacodylate buffer ( $\mathrm{pH} 7.4)$ containing

$6842.5 \%$ glutaraldehyde for 1 hour at room temperature. The bony capsule was dissected out and the

685 lateral wall, Reissner's membrane, and tectorial membrane were removed. The organ of Corti

686 was then dissected and fixed in a solution of $0.1 \mathrm{M}$ sodium cacodylate buffer $(\mathrm{pH} 7.4), 2 \mathrm{mM}$

687 calcium chloride, $2.5 \%$ glutaraldehyde, and 3.5\% sucrose for overnight at $4{ }^{\circ} \mathrm{C}$. Following

688 fixation, samples were prepared for SEM using the osmium tetroxide - thiocarbohydrazide

689 (OTOTO) method ${ }^{42}$ Then, the specimens were dehydrated in a graded ethanol solution, dried

690 using a critical point dryer (HCP-2, Hitachi, Japan), attached on the stub, and then coated with

691 platinum using a sputter coater (E1030, Hitachi, Japan). The surfaces of the organ of Corti were

692 captured under a cold-field emission SEM (SU8220, Hitachi, Japan) that was operated at 10 or

$69315 \mathrm{kV}$. Micrograph measurements were performed using ImageJ software (National Institutes of

694 Health, http://rsbweb.nih.gov/ij/), via the polygon tool for cell area measurements.

695

696 Bioinformatics. In silico prediction Algorithm: Polyphen-2

697 (http://genetics.bwh.harvard.edu/pph2/index.shtml) ; SIFT

698 (http://sift.jcvi.org/www/SIFT chr_coords submit.html); Conservation tools: GERP++ score in

699 the UCSC Genome Browser (http://genome-asia.ucsc.edu/) ; ExAC, Exome Aggregation

700 Consortium (http://exac.broadinstitute.org/) ;1000 Genomes

701 (https://www.ncbi.nlm.nih.gov/variation/tools/1000genomes/) ; KRGDB, Korean Reference 

'MEGA version 5' ${ }^{43}$.

Data analysis and statistical analysis. Off-line analysis was carried out using Clampfit version

$* * P<0.01, * * * P<0.001$ and $\# P<0.0001$.

\section{References:}

1. Zhao, H.B., Kikuchi, T., Ngezahayo, A. \& White, T.W. Gap junctions and cochlear homeostasis. J. Membr. Biol. 209, 177-186 (2006).

2. Wan, G., Corfas, G. \& Stone, J.S. Inner ear supporting cells: rethinking the silent majority. Semin Cell Dev Biol 24, 448-459 (2013).

3. Cohen-Salmon, M., et al. Targeted Ablation of Connexin26 in the Inner Ear Epithelial Gap Junction Network Causes Hearing Impairment and Cell Death. Current Biology 12, 1106-1111 (2002).

4. $\quad$ Erbe, C.B., Harris, K.C., Runge-Samuelson, C.L., Flanary, V.A. \& Wackym, P.A. Connexin 26 and Connexin 30 Mutations in Children with Nonsyndromic Hearing Loss. The Laryngoscope 114, 607-611 (2004).

5. Teubner, B. Connexin30 (Gjb6)-deficiency causes severe hearing impairment and lack of endocochlear potential. Human Molecular Genetics 12, 13-21 (2003).

6. Elzouki, A.Y., Harfi, H.A. \& Nazer, H.M. Textbook of clinical pediatrics, (Lippincott Williams \& Wilkins, Philadelphia, 2001). 
7. James, S.L., et al. Global, regional, and national incidence, prevalence, and years lived with disability for 354 diseases and injuries for 195 countries and territories, 1990-2017: a systematic analysis for the Global Burden of Disease Study 2017. The Lancet 392, 1789-1858 (2018).

8. Kim, N.K., et al. Whole-exome sequencing reveals diverse modes of inheritance in sporadic mild to moderate sensorineural hearing loss in a pediatric population. Genetics in medicine : official journal of the American College of Medical Genetics 17, 901-911 (2015).

9. Delmaghani, S., et al. Mutations in the gene encoding pejvakin, a newly identified protein of the afferent auditory pathway, cause DFNB59 auditory neuropathy. Nature genetics 38, 770-778 (2006).

10. Azaiez, H., et al. HOMER2, a stereociliary scaffolding protein, is essential for normal hearing in humans and mice. PLoS genetics 11, e1005137 (2015).

11. Kurima, K., et al. Dominant and recessive deafness caused by mutations of a novel gene, TMC1, required for cochlear hair-cell function. Nature genetics 30, 277-284 (2002).

12. Cheng, J., et al. Functional mutation of SMAC/DIABLO, encoding a mitochondrial proapoptotic protein, causes human progressive hearing loss DFNA64. American journal of human genetics 89, 56-66 (2011).

13. Yasunaga, S., et al. A mutation in OTOF, encoding otoferlin, a FER-1-like protein, causes DFNB9, a nonsyndromic form of deafness. Nature genetics 21, 363-369 (1999).

14. Schoen, C.J., et al. Increased activity of Diaphanous homolog 3 (DIAPH3)/diaphanous causes hearing defects in humans with auditory neuropathy and in Drosophila. Proceedings of the National Academy of Sciences of the United States of America 107, 13396-13401 (2010).

15. Ruel, J., et al. Impairment of SLC17A8 encoding vesicular glutamate transporter-3, VGLUT3, underlies nonsyndromic deafness DFNA25 and inner hair cell dysfunction in null mice. American journal of human genetics 83, 278-292 (2008).

16. Zong, L., et al. Mutations in apoptosis-inducing factor cause X-linked recessive auditory neuropathy spectrum disorder. Journal of medical genetics 52, 523-531 (2015).

17. Santarelli, R., et al. OPA1-related auditory neuropathy: site of lesion and outcome of cochlear implantation. Brain : a journal of neurology 138, 563-576 (2015).

18. Han, K.H., et al. ATP1A3 mutations can cause progressive auditory neuropathy: a new gene of auditory synaptopathy. Scientific reports 7, 16504 (2017).

19. Oza, A.M., et al. Expert specification of the ACMG/AMP variant interpretation guidelines for genetic hearing loss. Human mutation 39, 1593-1613 (2018).

20. Richards, S., et al. Standards and guidelines for the interpretation of sequence variants: a joint consensus recommendation of the American College of Medical Genetics and Genomics and the Association for Molecular Pathology. Genetics in medicine : official journal of the American College of Medical Genetics 17, 405-424 (2015).

21. Rentzsch P, W.D., Cooper GM, Shendure J, Kircher M. . CADD: predicting the deleteriousness of variants throughout the human genome. Nucleic Acids Research (2018).

22. Merner, N.D., et al. Arrhythmogenic right ventricular cardiomyopathy type 5 is a fully penetrant, lethal arrhythmic disorder caused by a missense mutation in the TMEM43 gene. American journal of human genetics 82, 809-821 (2008).

23. Siragam, V., et al. TMEM43 mutation p.S358L alters intercalated disc protein expression and reduces conduction velocity in arrhythmogenic right ventricular cardiomyopathy. PloS one 9, e109128 (2014).

24. Bengtsson, L. \& Otto, H. LUMA interacts with emerin and influences its distribution at the inner nuclear membrane. J Cell Sci 121, 536-548 (2008).

25. Strenzke, N., et al. Hair cell synaptic dysfunction, auditory fatigue and thermal sensitivity in otoferlin Ile515Thr mutants. The EMBO journal 35, 2519-2535 (2016).

26. Johnson, S.L., et al. Connexin-Mediated Signaling in Nonsensory Cells Is Crucial for the Development of Sensory Inner Hair Cells in the Mouse Cochlea. The Journal of neuroscience : the official journal of the Society for Neuroscience 37, 258-268 (2017).

27. Wang, H.C., et al. Spontaneous Activity of Cochlear Hair Cells Triggered by Fluid Secretion Mechanism in Adjacent Support Cells. Cell 163, 1348-1359 (2015).

28. Sackin, H. Mechanosensitive Channels. Annu. Rev. Physiol. 57, 333-353 (1995).

29. Entwisle, L.K., Warren, S.E. \& Messersmith, J.J. Cochlear Implantation for Children and Adults with Severe-to-Profound Hearing Loss. Seminars in hearing 39, 390-404 (2018).

30. Lee, D.S., et al. Cross-modal plasticity and cochlear implants. Nature 409, 149-150 (2001).

31. Lee, J.S., et al. PET evidence of neuroplasticity in adult auditory cortex of postlingual deafness. Journal of nuclear medicine : official publication, Society of Nuclear Medicine 44, 1435-1439 (2003). 
32. Jiang, C., et al. TMEM43/LUMA is a key signaling component mediating EGFR-induced NF-kappaB activation and tumor progression. Oncogene 36, 2813-2823 (2017).

33. Hwang, E.M., et al. A disulphide-linked heterodimer of TWIK-1 and TREK-1 mediates passive conductance in astrocytes. Nat Commun 5, 3227 (2014).

34. Guoqiang Wana, b., Gabriel Corfasa,b,c, Jennifer S. Stoned. Inner ear supporting cells: Rethinking the silent majority. Semin. Cell Dev. Biol. (2013).

35. Walz, W. Role of astrocytes in the clearance of excess extracellular potassium. Neurochemistry international 36, 291-300 (2000).

36. O'Connor, E.R. \& Kimelberg, H.K. Role of calcium in astrocyte volume regulation and in the release of ions and amino acids. The Journal of neuroscience : the official journal of the Society for Neuroscience 13, 2638-2650 (1993).

37. Olson, J.E., Evers, J.A. \& Holtzman, D. Astrocyte volume regulation and ATP and phosphocreatine concentrations after exposure to salicylate, ammonium, and fatty acids. Metabolic brain disease 7, 183-196 (1992).

38. Nin, F., et al. The endocochlear potential depends on two K+ diffusion potentials and an electrical barrier in the stria vascularis of the inner ear. Proc. Natl. Acad. Sci. U. S. A. 105, 1751-1756 (2008).

39. Forester, B.P., et al. Age-related changes in brain energetics and phospholipid metabolism. NMR in biomedicine 23, 242-250 (2010).

40. Sang, S., et al. Proband Whole-Exome Sequencing Identified Genes Responsible for Autosomal Recessive Non-Syndromic Hearing Loss in 33 Chinese Nuclear Families. Frontiers in genetics 10, 639 (2019).

41. Ventura, A., et al. Cre-lox-regulated conditional RNA interference from transgenes. Proceedings of the National Academy of Sciences of the United States of America 101, 10380-10385 (2004).

42. Kim, M.A., et al. Methionine Sulfoxide Reductase B3-Targeted In Utero Gene Therapy Rescues Hearing Function in a Mouse Model of Congenital Sensorineural Hearing Loss. Antioxid Redox Signal 24, 590-602 (2016).

43. Tamura K, P.D., Peterson N, Stecher G, Nei M, and Kumar S. MEGA5: Molecular Evolutionary Genetics Analysis using Maximum Likelihood, Evolutionary Distance, and Maximum Parsimony Methods. Molecular Biology and Evolution. (2011).

\section{Acknowledgments}

Authors thank Dr. Young-Woo Seo at Korea Basic Science Institute Gwang-Ju Center for his assistance in image analysis with Imaris software. This research was supported by a grant of the Korea Health Technology R\&D Project through the Korea Health Industry Development Institute (KHIDI), funded by the Ministry of Health \& Welfare, Republic of Korea (grant number: HI15C1632, and HI17C0952 to B.Y. Choi), Creative Research Initiative Program, Korean National Research Foundation (2015R1A3A2066619 to C.J. Lee) and the National Research Council of Science \& Technology (NST) grant by the Korea government (No. CRC15-04-KIST to S.J. Oh).

\section{Author contributions}


H.R.P., J.W.K., S.H.O. and B.Y.C. recruited the family and provided samples. M.W.S. and X.L. did linkage analysis. N.K., J.L. and W.P. contributed to NGS. A.R.K., H.C. and X.L. did Sanger sequencing \& NGS results analysis. J.H.H., S.S., X.C. performed Sanger sequencing from additionally recruited family members. M.Y.K., S.B. and Z-Y.C. maintained cell line and IHC. M.W.L. and H.W.S. performed RT-PCR and initial experiments on trafficking of our target

834 protein in cell lines. J.B.S. made videos for localization of our target protein and also performed IHC of the mouse cochlea. J.J.H., B.J.K., D.H.W., E.Y. and K.S. performed dissection and IHC of the monkey cochlea. E.Y. and K.S. also contributed to making mouse cochlea culture, infecting it with TMEM43 shRNA and performing electrophysiology. K.Y.L., M.A.K., U.K.K. did SEM experiments. M.W.J. contributed to electro-physiology experiments, developed shRNA to knockdown Tmem43 and did immunocytochemistry for TMEM43 expressed cells. S.J.O. organization of the data into figures. J.J.H. also performed audiograms and speech supervised the project.

Figure 1 | Pedigree and audiological assessment of family HN66 and SB162. 
are superscripted. b, PTA, SDS, ABR and DPOAE of 6 subjects from HN66 and SB162. Unaffected subject \#290 shows a definite normal ABR response to sound stimulus as low as 40dB. Despite absence of ABR response, affected subjects (\#17, \#291, \#284, \#304) show either presence of DPOAE response (blue line) or cochlear microphonics (CM) (asterisk), indicating ANSD from these subjects. The youngest subject (\#36 from HN66, a 16-year-old female) among all affected members from the two families showed obviously disordered waveform differentiation in ABR responses and significant elevation of ABR threshold up to as high as $80 \mathrm{~dB}$, while DPOAE response of \#36 was completely preserved. This suggests that phenotype for \#36 can be interpreted as the stage of transition to canonical ANSD.

Figure 2 | A p.R372X variant of TMEM43 is the causative variant of hearing loss in family HN66 and SB162.

a, Overall workflow of variant detecting in families HN66 and SB162. b, Merlin multipoint linkage analysis demonstrates LOD scores greater than 3.0 only on chromosome 3p25.1. Region $\# 1$ (Chr. 3: 1,946,000 - 5,956,000), region \#2 (Chr. 3:11,883,000-14,502,000). c, Genetic candidates of the ANSD after exome sequencing of genomic DNA from 3 subjects ( 2 affected subjects (\#36 and \#28) and 1 unaffected subject (\#22)) in HN66 and 4 subjects (the 3 affected $(\# 289, \# 291, \# 284)$ and 1 unaffected, (\#290)) in SB162 are listed. Subsequent segregation study with additional subjects to identify TMEM43 in HN66 and SB162 as the only remaining candidate with full-match. Co-segregation of a gene variant with the hearing loss phenotype is indicated as green and an apricot colored box, while mismatch is shown as a white box. Chr; chromosome, AA; amino acid. d, DNA sequence chromatograms of c.1114C $>$ T variant in TMEM43. e, The mutation spectrum of TMEM43 associated with ARVD5 and EDMD7 reported 
in HGMD database (Top, black letter) and the variants identified in the present study (Bottom, p.R372X). The conservative prediction of the amino acid sequences of TMEM43 is shown below. Asterisks indicate the amino acids that are identical across all species. Three-dimensional modeling of wild-type human TMEM43 and the variant TMEM43, lacking TM4, identified in the present study. TM, transmembrane domain.

\section{Figure 3 | TMEM43 is exclusively expressed in the GLSs of mouse cochlea.}

a, RT-PCR gel analysis of TMEM43 in various regions of mouse. Arrow indicates TMEM43 expression (size 196bp) (Co, cochlea; He, heart; Ey, eye; Br, brain; Ki, kidney; Li, liver; NC, negative control). b,c Low magnification (b) and high magnification (c) confocal micrographs of cochlear turns with anti-TMEM43 $\left(\mathrm{Ab}_{1-1}\right)$ (green) and anti-calretinin (red). d, Spatiotemporal expression of TMEM43 (red) and phalloidin (green) at the mouse organ of Corti. TMEM43 immunoreactivity detected widely in the Kolliker's organ at P4 becomes restricted in the course of postnatal development at inner border cells and cell junctions of the inner sulcus cells at P20. Boxed area indicates magnified view of TMEM43 localization at the apical membrane or cortex of the inner border cell. e, Frozen-section images of the mouse cochlea at P6 immunolabeled by TMEM43 $\left(\mathrm{Ab}_{1-1}\right)$ (green) and counterstained by phalloidin (red). TMEM43 immunoreactivity is detected mainly at the organ of Corti and the magnified image shows TMEM43 immunoreactivity exclusively at the GLSs of the organ of Corti, not in the hair cells. f-i, In situ hybridization with a scrambled probe (f), with Tmem 43 probe showing Tmem 43 mRNA expression in the Organ of corti $(*)$ but not in the spiral ganglion neuron $(* *)$ and cochlear nerve $\left({ }^{* * *}\right)(\mathbf{g})$, with hair cell marker SMPX (h), and data with higher magnification (i). IHC; Inner hair cell, OHC; Outer hair cell, IBC; Inner border cell, BC; border cell, DC; Deiters cells, RM; 
Reisners membrane.

\section{Figure 4 | p.R372X of TMEM43 shows decreased cell surface expression with reduced}

\section{protein stability.}

a, 2D topology of TMEM43 WT and p.R372X. Antibody epitopes are indicated as Ab $\mathrm{b}_{1-1}$ and $\mathrm{Ab}_{1-2}$ and FLAG is tagged at C-terminal. b, Immunostaining result of TMEM43 expressing HEK293T cells with and without cell permeabilization. Cells were double stained with FLAG and TMEM43 antibody $\left(\mathrm{Ab}_{1-2}\right)$. c, Cell surface biotinylation assay in TMEM43 WT and p.R372X transfected HEK293T cells blotted with TMEM43 antibody $\left(\mathrm{Ab}_{1-2}\right)$. d, Cycloheximide (CHX) assay with FLAG antibody. $40 \mathrm{ug} / \mathrm{ml} \mathrm{CHX} \mathrm{was} \mathrm{used.} \mathrm{Band} \mathrm{sizes} \mathrm{for} \mathrm{TMEM43} \mathrm{WT} \mathrm{and}$ TMEM43-p.R372X are 44kD and 41kD each.

\section{Figure 5 | TMEM43-p.R372X KI mice display morphological and physiological}

\section{dysfunction.}

a, Schematic flow of generation of Tmem43-p.R372X KI (C57BL/6J;129S-Tmem43 ${ }^{\text {tm ICby }}$ ) mice model using Clustered Regularly Interspaced Short Palindromic Repeats (CRISPR) technology. Double stop codons were inserted at p.R372. A germline transmission of p.R372X was confirmed. The p.R372X site, KI-targeting shRNA sequence and restriction enzyme site of BsiEI are each noted with red, green and blue color. Genotyping result using BsiEI is shown. gRNA: guide RNA, ssODN: Single-stranded oligodeoxynucleotide donor, HR: homologous recombination. $\mathbf{b}$, Confocal micrographs of the organs of Corti from age-matched (1 month old) littermates of $T m e m 43^{+/+}, T m e m 43^{+/ K I}$ and $T m e m 43^{K I / K I} .3 \mathrm{D}$ reconstruction images were obtained from the apical turn of cochlea immunolabeled with anti-TMEM43 $\left(\mathrm{Ab}_{1-1}\right)(\mathrm{red})$ and anti- 
921

922

923

924

925

926

927

928

929

930

931

932

933

934

935

936

937

938

939

940

941

942

943

calretinin (green). XZ and YZ section views (third row) and surface object clipping images of a single IHC (fourth row) show that TMEM43 immunoreactivity is found primarily in the apical potion of inner border cell, juxtaposing the upper lateral membrane of calretinin-positive IHC. c, SEM findings at the level of reticular lamina of hair cells. Green and light green dotted line each indicates apical surface area of inner border cells of $T m e m 43^{+/+}$and $T m e m 43^{+/ K I}$ respectively. d, Summary graphs of apical surface area of inner border cells $(\mathrm{n}=50)$ from $(\mathbf{c})$. e, Auditory brainstem response (ABR) waveforms recorded in $T m e m 43^{+/+}$and $T m e m 43^{+/ K I}$ mice at 3,6 , and 9 months. The $\mathrm{x}$ axis indicates the different time, and the $\mathrm{y}$ axis indicates the sound pressure level (SPL). 6 and 9 months Tmem $43^{+/ K I}$ mice show no detectable wave response to 50 and $60 \mathrm{~dB}$ tone burst sound in ABR, in contrast to the $T m e m 43^{+/+}$mice, indicative of progressive deafness. f, Mean \pm SEM ABR thresholds from littermate $T m e m 43^{+/+}$(red, $\mathrm{n}=3$ ), Tmem $43^{+/ K I}$ (green, $\mathrm{n}=12$ ), and Tmem $43^{K I / K I}$ (blue, $\mathrm{n}=3$ ) measured at 6 months after birth. $\mathbf{g}$, Mean \pm SEM ABR wave I amplitude growth function for littermate $\operatorname{Tmem}_{43^{+/+}}(\mathrm{red}, \mathrm{n}=4), \operatorname{Tmem} 43^{+/ K I}$ (green, $\left.\mathrm{n}=4\right)$ and Tmem $43^{K I / K I}$ (blue, $\mathrm{n}=4$ ) at $8 \mathrm{kHz}$ measured at 6 months after birth. h, Mean $\pm \mathrm{SEM}$ ABR wave I amplitude growth function for Tmem $43^{+/+}$(blue, $\left.\mathrm{n}=3\right), \operatorname{Tmem}_{4} 3^{+/ K I}(\mathrm{red}, \mathrm{n}=3)$ and $T m e m 43^{K I / K I}$ (green, $\mathrm{n}=3$ ) at $8 \mathrm{kHz}$ measured at 3 months after birth. ABR wave I amplitudes were not significantly different among each genotype at whole decibels measured.

\section{Figure 6 | TMEM43 is an essential component of connexin channels to induce passive}

\section{conductance current in GLSs.}

a, Immunohistochemistry data showing TMEM43 expression with Cx26 (upper panel), and with Cx30 (bottom panel) in GLSs (P6). b, Confocal micrographs of Cx26 expression in Organ of
Corti (upper panel) and Stria Vascularis (bottom panel) of $T m e m 43^{+/+}, \operatorname{Tmem}_{4} 3^{+/ K I}$ and 
$944 T$ Tmem $43^{K I / K I}$ (5 month). There was no difference among the genotypes. c, Co-IP data show that both TMEM43 WT and TMEM43-p.R372X are immuno-pulled down with Cx26 and Cx30.

946

947

948

949

950

951

952

953

954

955

956

957

958

959

960

961

962

963

964

965

966

d,f,h Representative I-V curve measured from GLSs of control (black), carbenoxolone treated (d), $\mathrm{GdCl}_{3}$ treated (f) and pH6 treated (h) (red) cochlea. e,g,i Summary bar graph of $\mathbf{d}(\mathbf{e}), \mathbf{f}(\mathbf{g})$ and h (i). Glass pipette was filled with K-Gluconate internal solution. j, Representative I-V curve measured from naïve (black), control shRNA treated (green) and Tmem 43 shRNA treated (red) cochlea. k, Summary bar graph of (j). l, Representative current-voltage relationship of Tmem $43^{+/+}$(red), Tmem $43^{+/ K I}$ (green) and Tmem $43^{K I / K I}$ (blue). m, Summary bar graph of GLS currents from (l). n-p, GLS current measured from Tmem43 KI shRNA un-infected and infected cochlea of Tmem $43^{+/+}(\mathbf{n}), \operatorname{Tmem} 43^{+/ K I}(\mathbf{o})$ and $T m e m 43^{K I / K I}(\mathbf{p})$ mice. Note that the Tmem43 KI shRNA rescues impaired passive current of $T m e m 43^{+/ K I}$. q, Summary bar graph of (n-p).

\section{Figure 7 | Timely cochlea implantations in family SB162 rescue impaired speech}

\section{discrimination.}

a, Speech discrimination performance scores of subject 284 measured at 3, 6 and 12 months following cochlear implantation. Telephone speech discrimination was $100 \%$ at 12 months following cochlea implantation. $\mathbf{b}$, Longitudinal change of postoperative auditory performance from control subjects $(n=39)$ with adult onset progressive hearing loss: Scores at postoperative 3 months, 6 months, 1year as measured by K-CID, Spondee and PB word are shown. c, Subject 304 showed improved K-CID (up to 22.2\%) and PB word (up to 4\%) score after cochlear implantation. KCID Sentence score: Korean Central Institute for the Deaf score. PB word score: phonetically balanced word score. Spondee words: audiology a word with 2 syllables-disyllabic, that is pronounced with equal emphasis the $1^{\text {st }}$ and $2^{\text {nd }}$ syllables. d, The responses of Electrical 
967 Auditory Brainstem Responses (e-ABR) obtained through the cochlear implant electrodes

968 inserted in the right ear (shown in red) are evident at the entire channel tested (No. 3, 5, 8) in

969 subject 304. Conventionally, the responses of the right and left ear are shown in red and blue,

970 respectively. The wave V (annotated on the waves) was detected at about $4 \mathrm{~ms}$ after electrical

971 stimulation and the thresholds was ranged from 500 to $800 \mu \mathrm{V}$. e, The results of e-ABR obtained

972 through the electrodes implanted in the left ear of the representative case of postsynaptic auditory

973 neuropathy spectrum disorder are shown in blue. The wave V was not detected even with the

974 maximum intensity of stimulation $(1000 \mu \mathrm{v})$ due to the dys-synchronic response of cochlear

975 nerve. 
bioRxiv preprint doi: https://doi.org/10.1101/2020.07.27.222323; this version posted July 27, 2020. The copyright holder for this preprint (which Figure 1
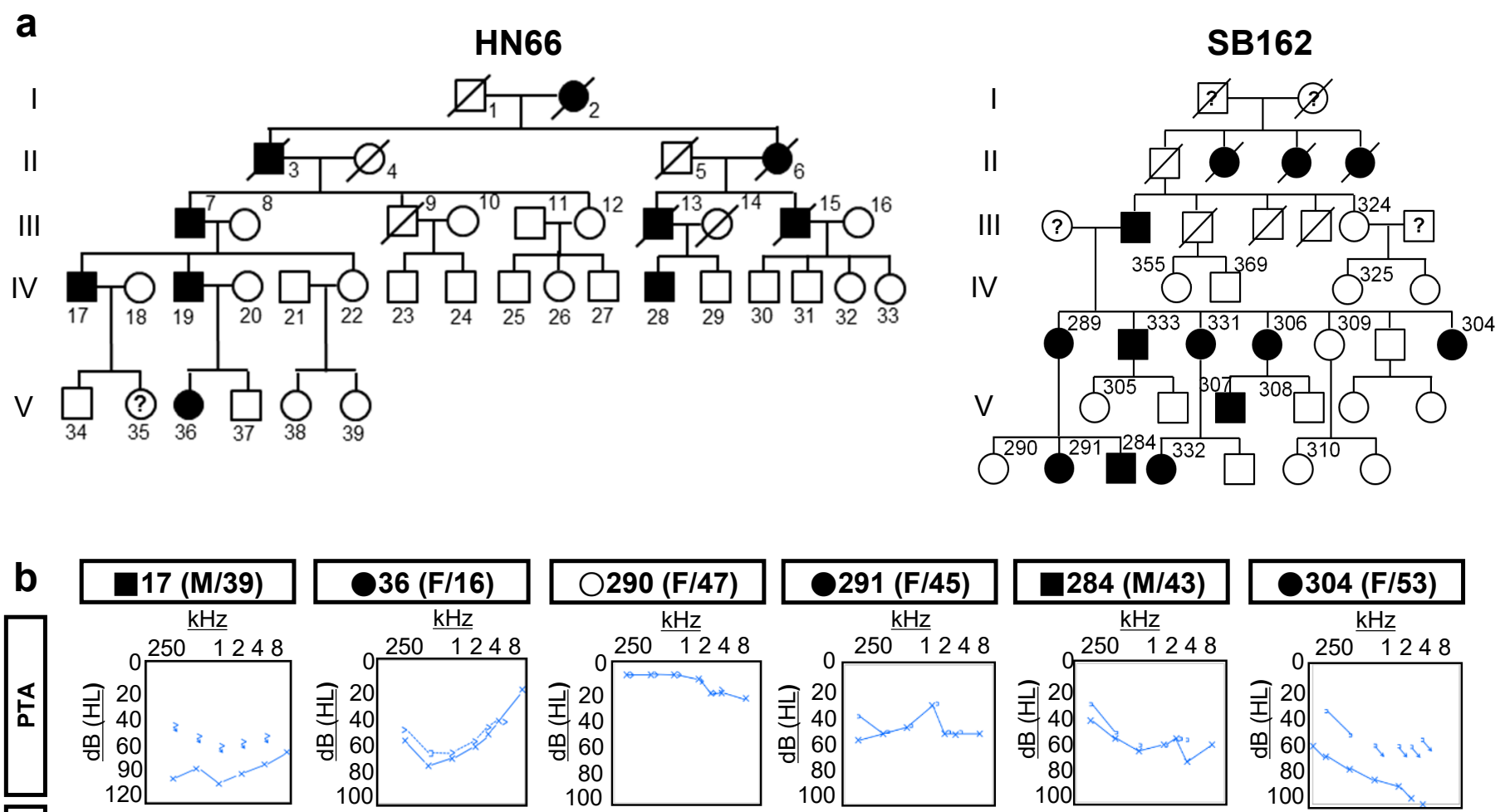

ณ

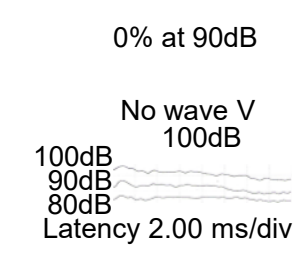

$$
50 \% \text { at } 90 \mathrm{~dB}
$$

$100 \%$ at $42 \mathrm{~dB}$

$4 \%$ at $86 \mathrm{~dB}$

$32 \%$ at $88 \mathrm{~dB}$

$0 \%$ at $90 \mathrm{~dB}$
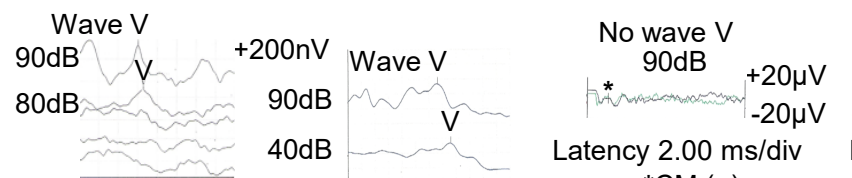

$\begin{gathered}\text { No wave } \mathrm{V} \\ 90 \mathrm{~dB}\end{gathered}$
$+20 \mu \mathrm{V}$
$-20 \mu \mathrm{V}$

No wave $\mathrm{V}$

Latency $2.00 \mathrm{~ms} / \mathrm{div}$

${ }^{*} \mathrm{CM}(+)$

No CM

90dB
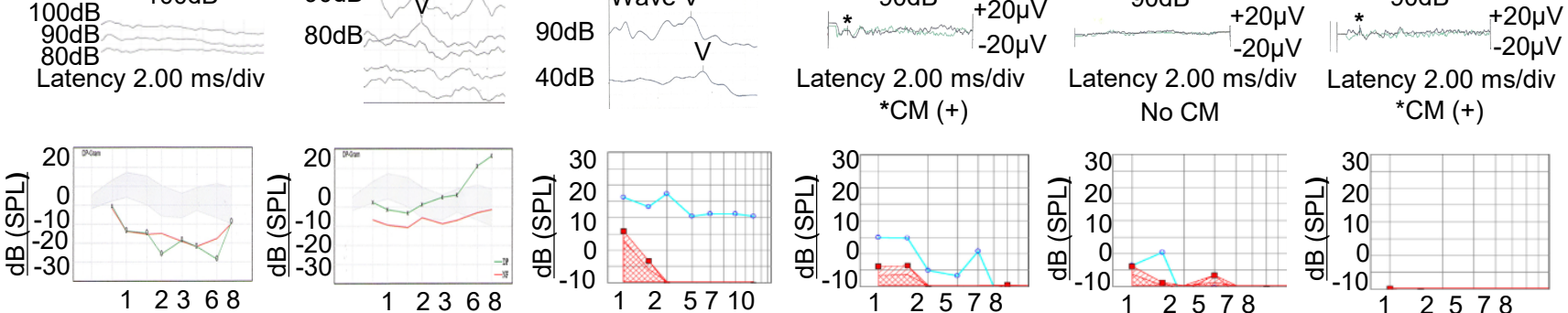

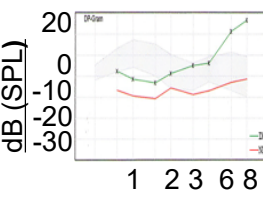
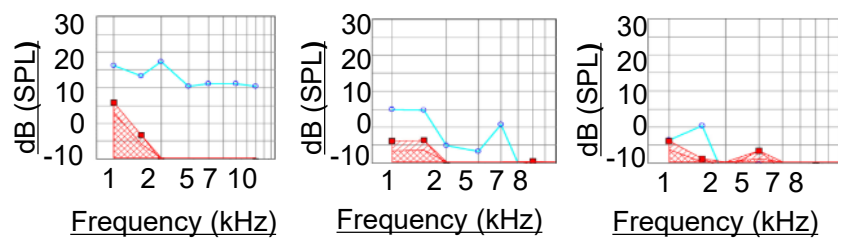

${ }^{*} \mathrm{CM}(+)$

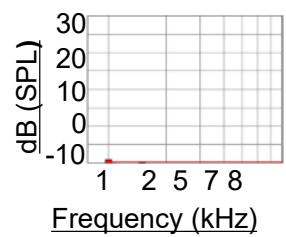


bioRxiv preprint doi: https://doi.org/10.1101/2020.07.27.222323; this version posted July 27, 2020. The copyright holder for this preprint (which Figure 2

a

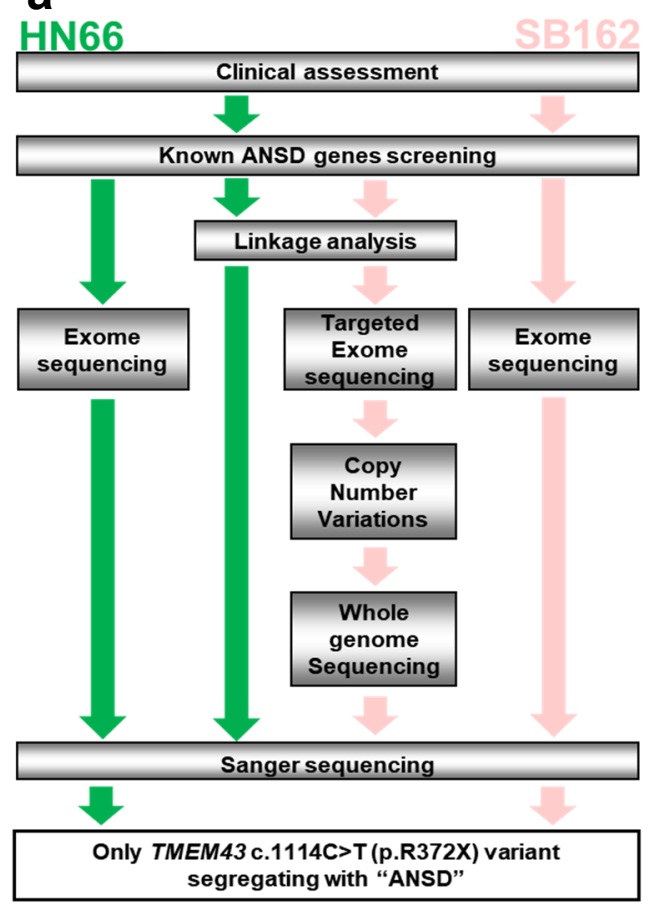

d $\quad$ HN66

TMEM43

c.1114C>T;p.R372X

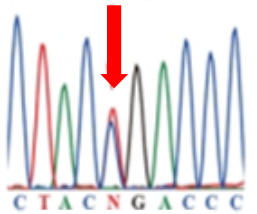

Wildtype

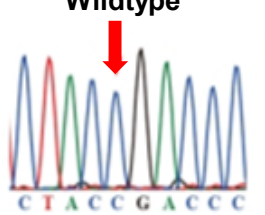

C I ACC GACCC b

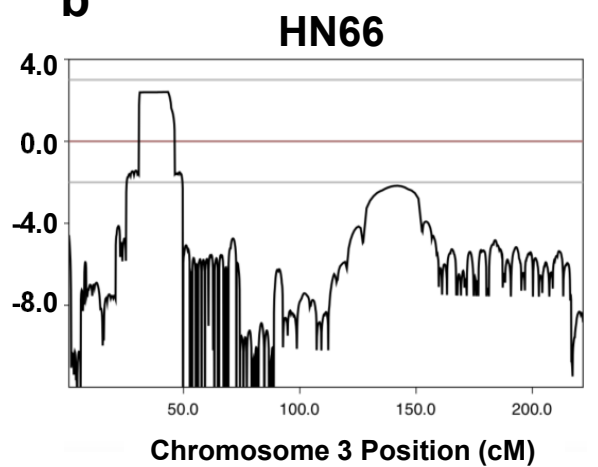

Chromosome 3 Position (cM)

$\mathbf{C}$

\begin{tabular}{|c|c|c|c|c|c|c|c|c|c|c|c|c|c|c|c|}
\hline \multirow[t]{2}{*}{ Gene } & \multirow[t]{2}{*}{$\mathrm{Chr}$} & \multirow[t]{2}{*}{ Nucleotide } & \multirow[t]{2}{*}{ AA } & 7 & 17 & 19 & 28 & \begin{tabular}{|l|l}
36 & 1
\end{tabular} & \begin{tabular}{l|l}
12 & 22
\end{tabular} & \begin{tabular}{l|l}
22 & 23
\end{tabular} & \begin{tabular}{l|l}
33 & 24
\end{tabular} & \begin{tabular}{l|l}
4 & 25
\end{tabular} & 29 & 37 & 38 \\
\hline & & & & - & a & a & a & $\bullet$ & \begin{tabular}{l|l}
0 & 0 \\
0
\end{tabular} & \begin{tabular}{l|l}
0 & 5
\end{tabular} & $\square$ & a & $\square$ & $\square$ & 0 \\
\hline TMEM43 & $\mathrm{chr} 3$ & c.C1114T & p. $R 372 X$ & & & & & & & & & & & & \\
\hline CTBP2 & chr10 & c. T2315G & p. L L772W & & & & & & & & & & & & \\
\hline VILL & chr3 & c.946detT & p.F316fs & & & & & & & & & & & & \\
\hline XIRP1 & chr3 & c. $T 281 \mathrm{C}$ & p.M94T & & & & & & & & & & & & \\
\hline CHODL & chr21 & c. $\mathrm{T665 \textrm {C }}$ & p. $1222 T$ & & & & & & & & & & & & \\
\hline
\end{tabular}

SB162

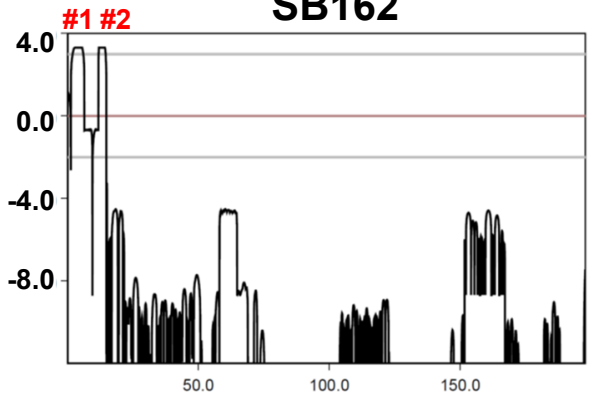

Chromosome 3 Position (cM)

SB162

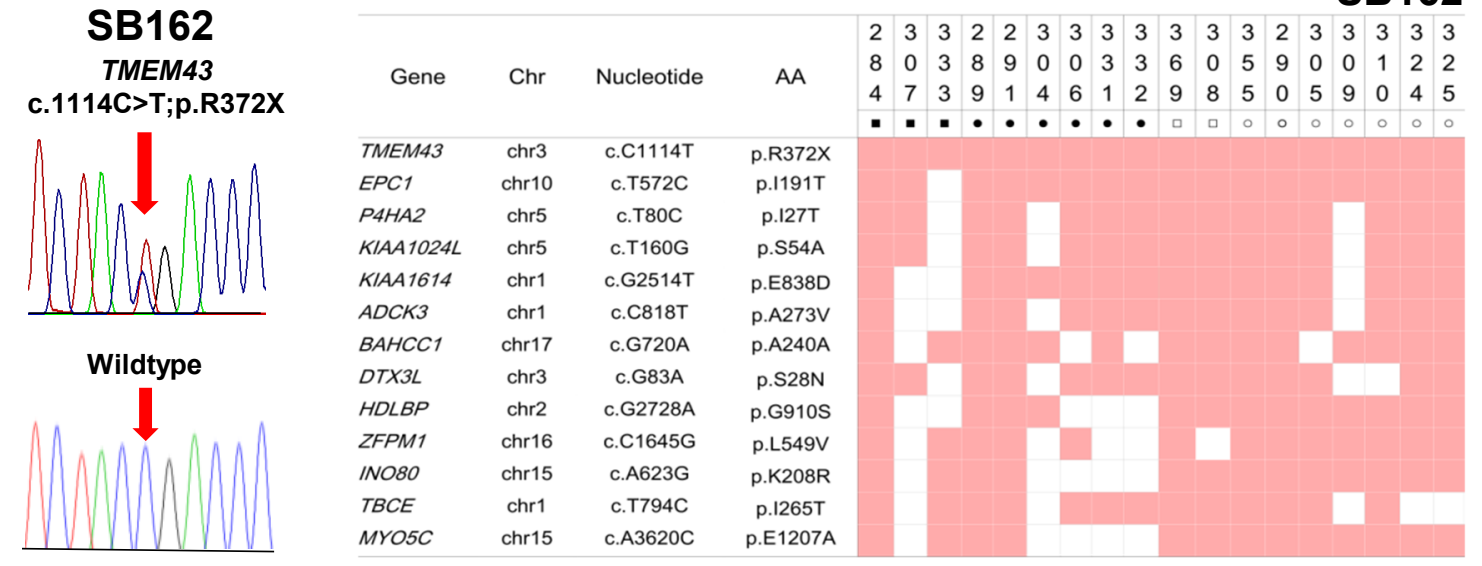

e

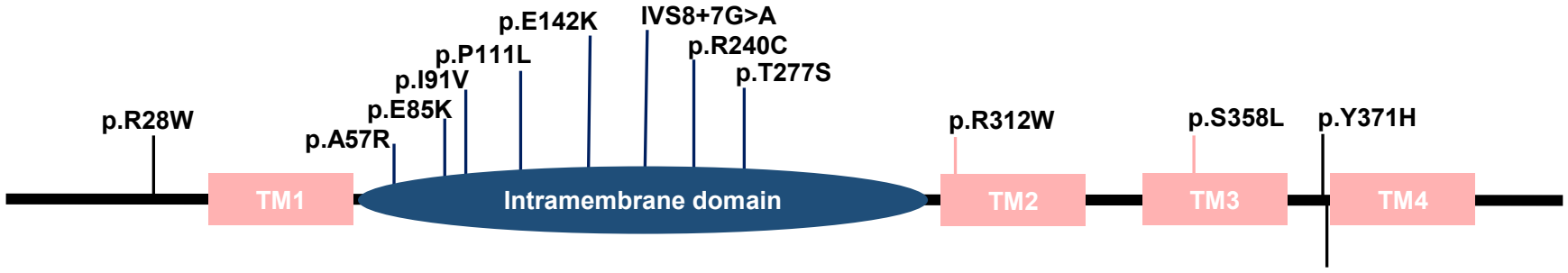

\section{Loop2 TM3 Loop3R372X TM4}

Pogona vitticeps WFPVVRDLVNIGLKAFAFCLATSLSLLTISVGWLFPPLWAILIGLLAA-VPIVLARSRV Beluga whale WFPIFRDLVN IGLKAFALCVATSLTLLTVAAGWLFHPLWALVISCLAL-VPI IVARTRV Canis lupus familiaris WFPIFRDLVDIGLKAFAFCVATSLTLLTVAAGWLFPPLCALFISCLAL-VPIIIARTRV Bos Taurus WFPVFRDLVN I GVKAFAFCVATSLTLLTVAGGLFY PLWALGIAGLAL-VP I IARTRV Homo sapiens WFPVFRDLVN I GLKAFAFCVATSLTLLTVAAGMLFP PLWALLIAGLAL-VPILVARTRV Mus musculus WFPVFRDLVN IGLKAFAFCVATSLTLLTVAAGWLFYPLWAAL IGCLAL-VP I IARTRV Rattus norvegicus WFPVFRDLVN IGL KAFAFCVATSLTLLTVAAGIFYP PLWAALLGCLAL-VP I IARTRV Danio rerio RVPLLGGLLSVGLQLFCLCSSVSLSLLTIAAGN IYYPLLALCVAASALVLPLALAHARA Astyanax mexicanus WVPVRELVSVGLKVFALCVSCSLSLLTIAVGWLFYBLLAGLISALAL-VPILIARSRA

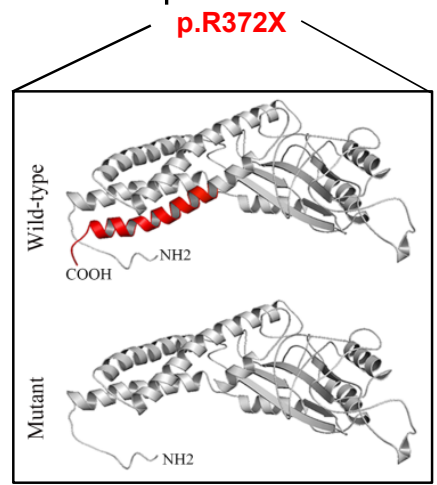


bioRxiv preprint doi: https://doi.org/10.1101/2020.07.27.222323; this version posted July 27, 2020. The copyright holder for this preprint (which Figure 3
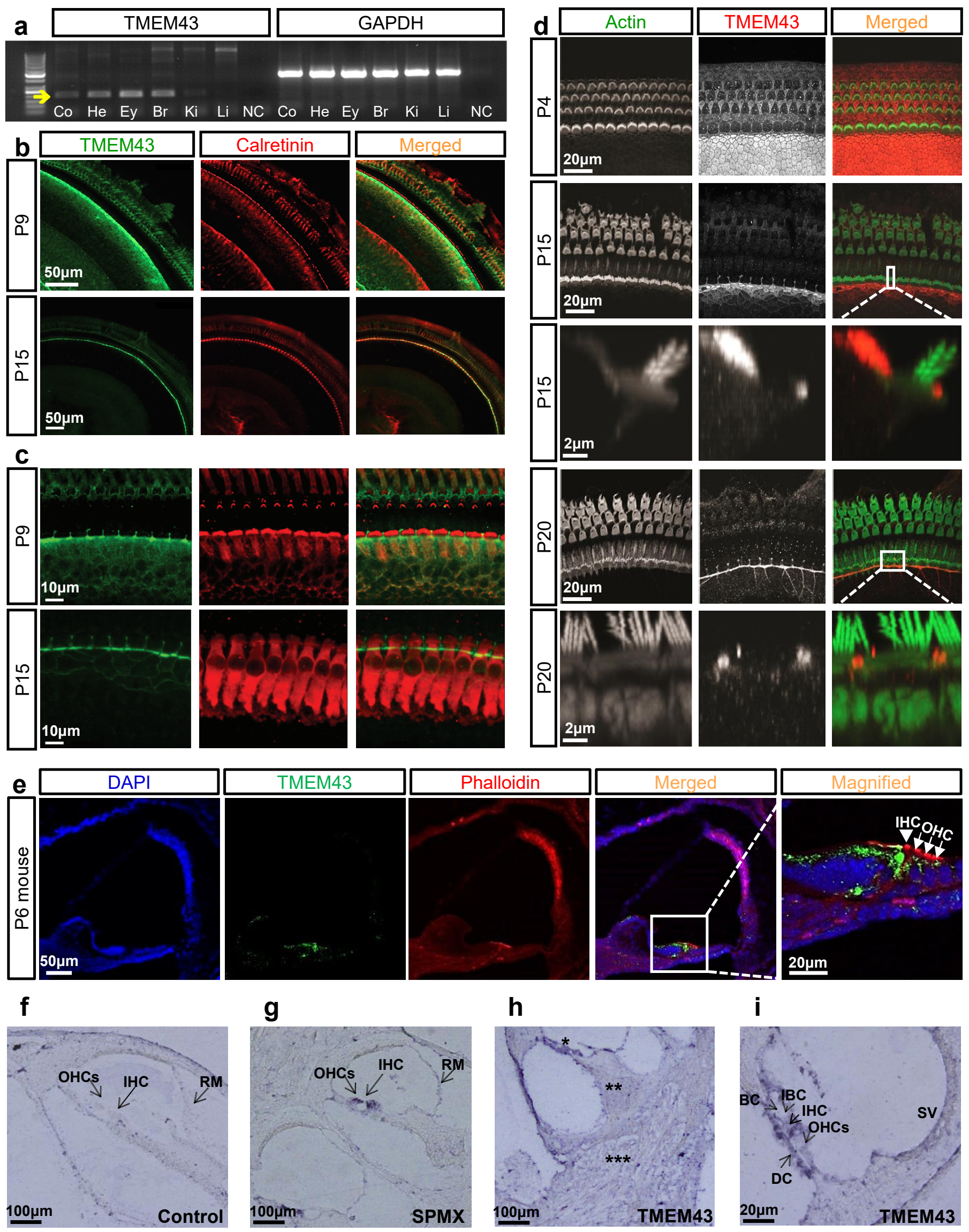

\section{h}

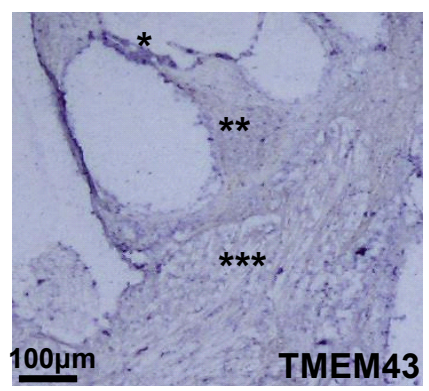

\section{i}

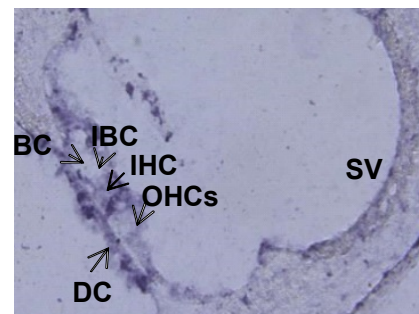

$20 \mu \mathrm{m}$

DC

TMEM43
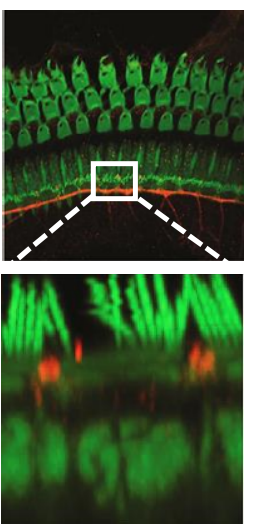

$20 \mu \mathrm{m}$ 
bioRxiv preprint doi: https://doi.org/10.1101/2020.07.27.222323; this version posted July 27, 2020. The copyright holder for this preprint (which Figure 4

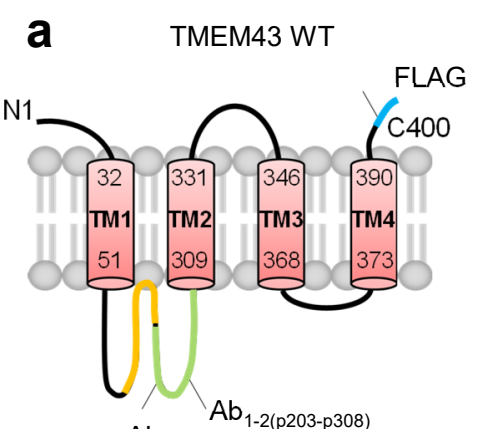

$A b_{1-1(p 100-p 200)}$
TMEM43 p.R372X

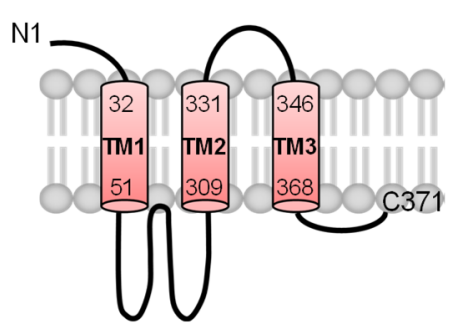

C

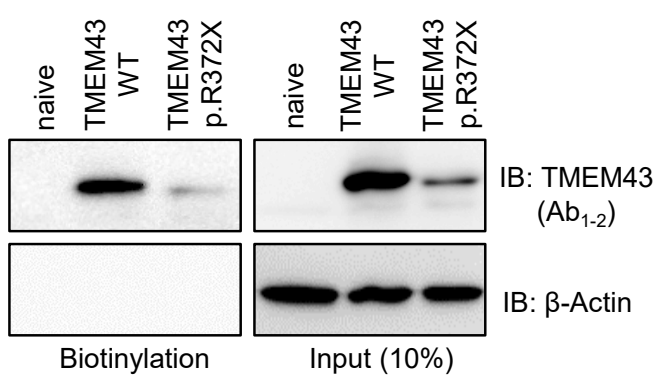

b

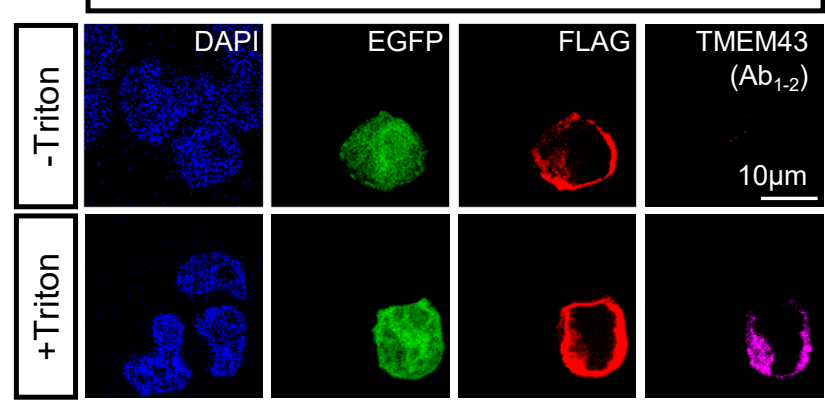

d

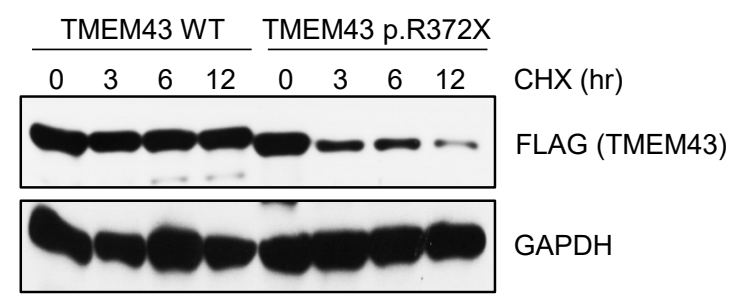


bioRxiv preprint doi: https://doi.org/10.1101/2020.07.27.222323; this version posted July 27, 2020. The copyright holder for this preprint (which Figure 5

a

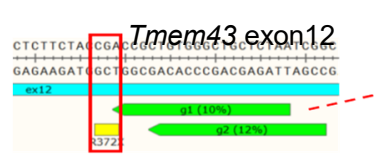

WT: Tmem43 $3^{+/+}$

Tmem43+/tm1cby: Tmem43+/KI

Tmem43 ${ }^{\text {tm1cby/tm1cby: Tmem43 }}{ }^{K I / K I}$
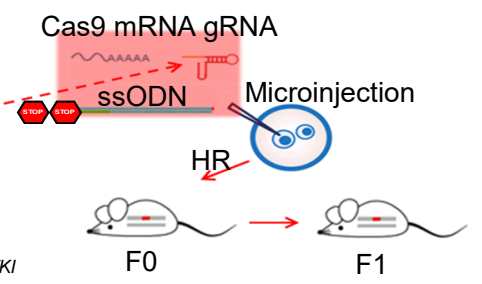

Reference p.R372

CTGCTGGCTGGCTCTTCTACCGACCGCTGTG WT/WT
CTGCTGGCTGGCTCtTCtad CGACG BsiEl CTGCTGGCTGGCTCTTCTACCACCG ATG

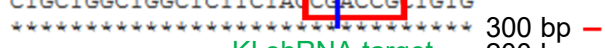
KI shRNA target $200 \mathrm{bp}$ CTGCTGGCtgGCtcttctad TGATGAtatg $100 \mathrm{bp}-$ CTGCTGGCTGGCTCTTCTAdTGATGA FTG-

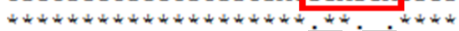
STOPSTOP

c
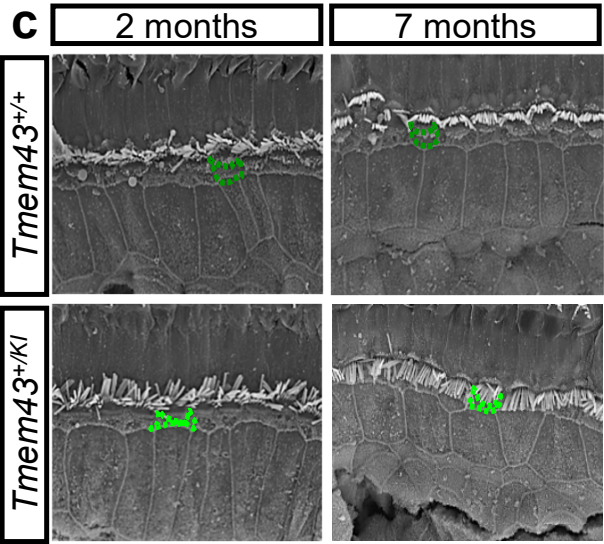

d
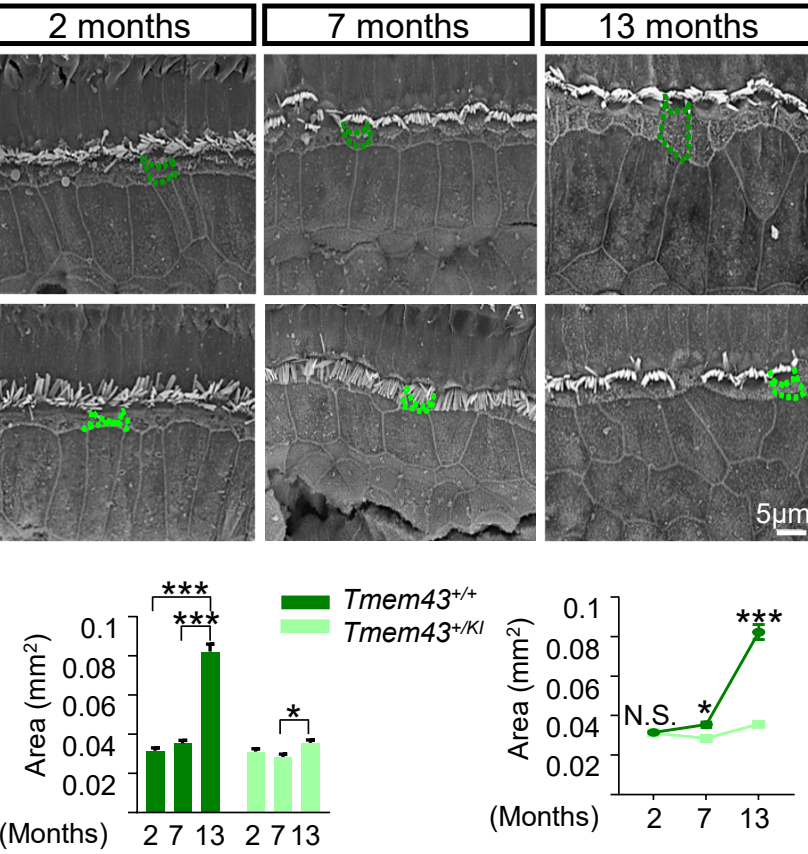

5um

f 6 months old

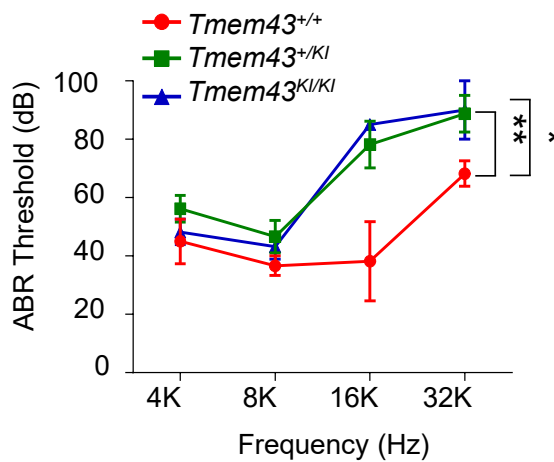

g 6 months old $(8 \mathrm{kHz})$

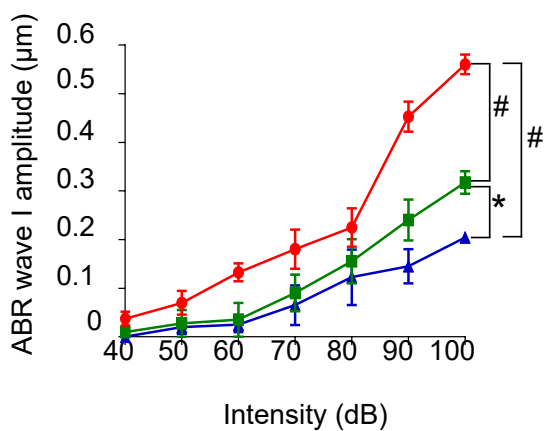

h $\quad 3$ months old $(8 \mathrm{kHz})$

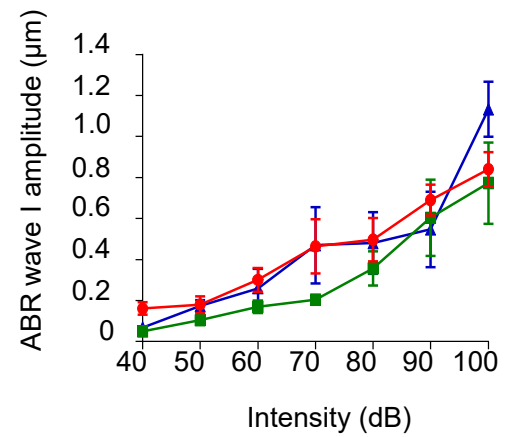


bioRxiv preprint doi: https://doi.org/10.1101/2020.07.27.222323; this version posted July 27, 2020. The copyright holder for this preprint (which Figure 6

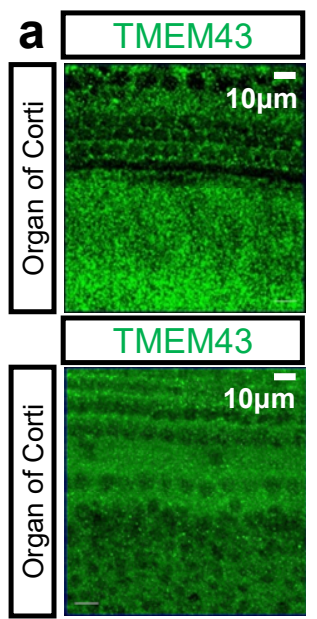

C
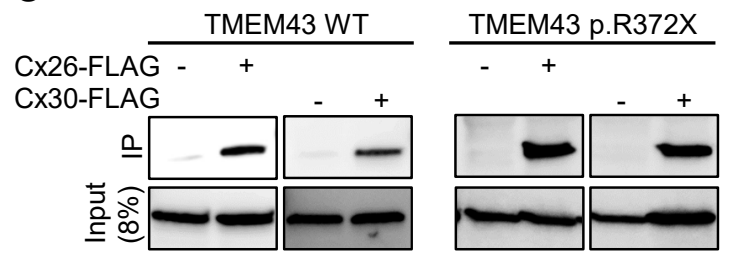

IP: FLAG

f
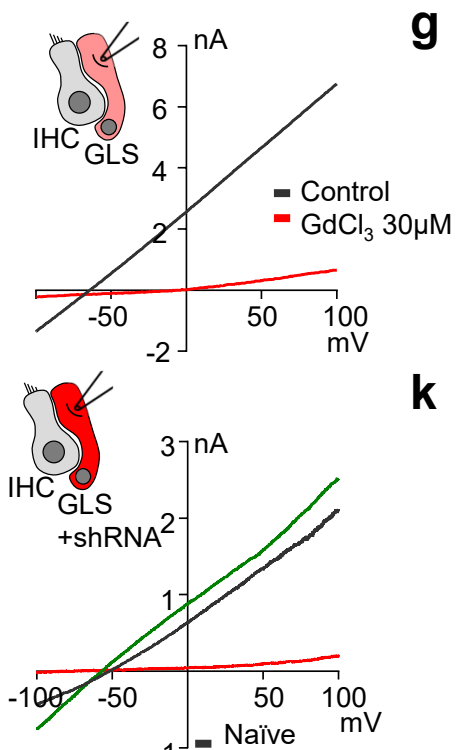

$-1=$ Naïve $\quad$ Control shNRA

- Tmem43 shRNA

n

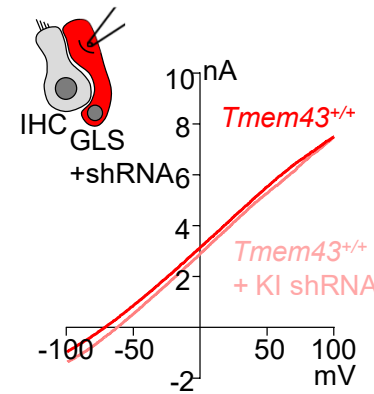

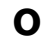

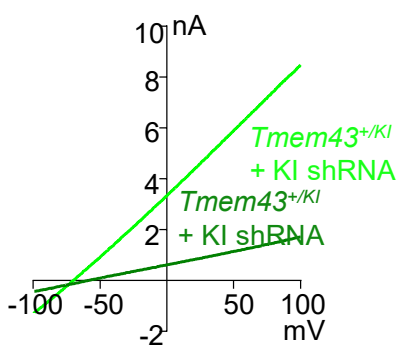

k
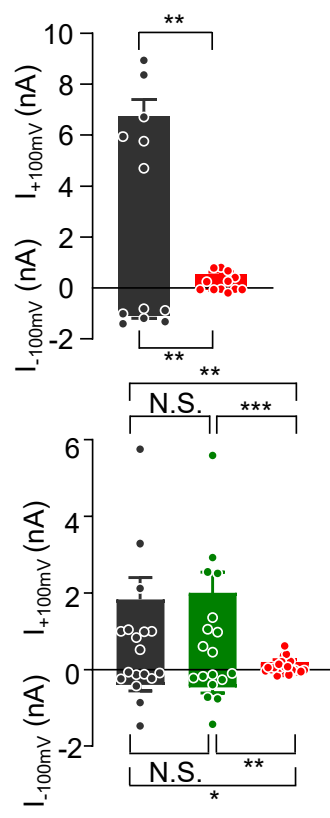
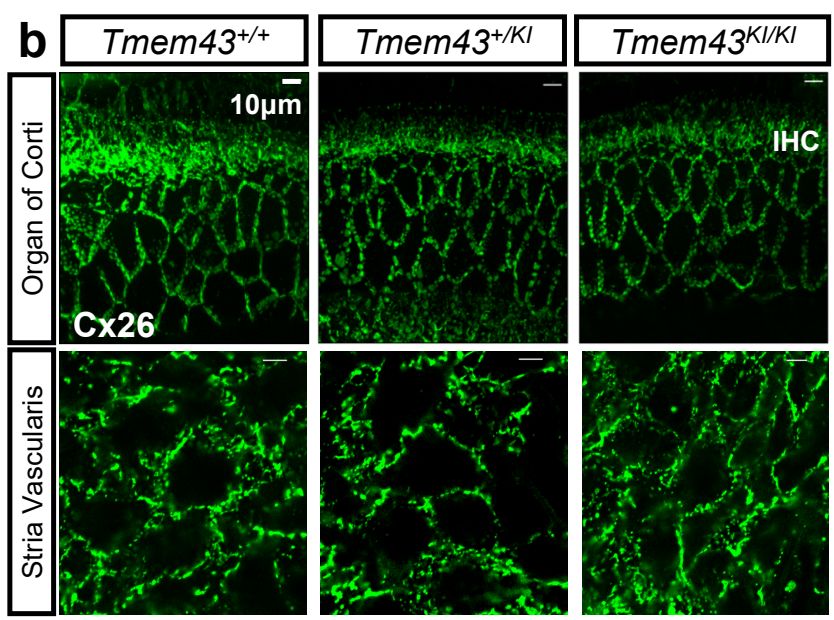

d

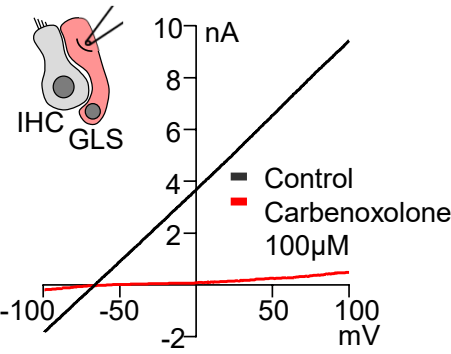

h

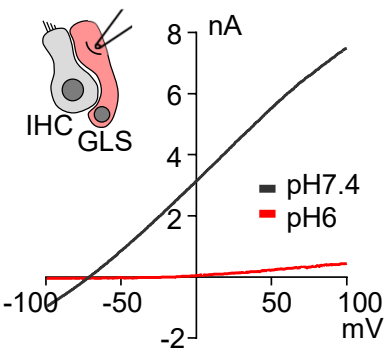

I

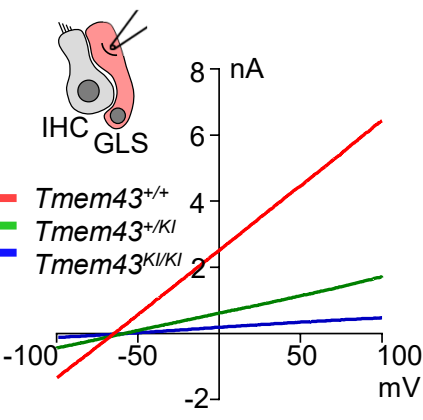

m

e
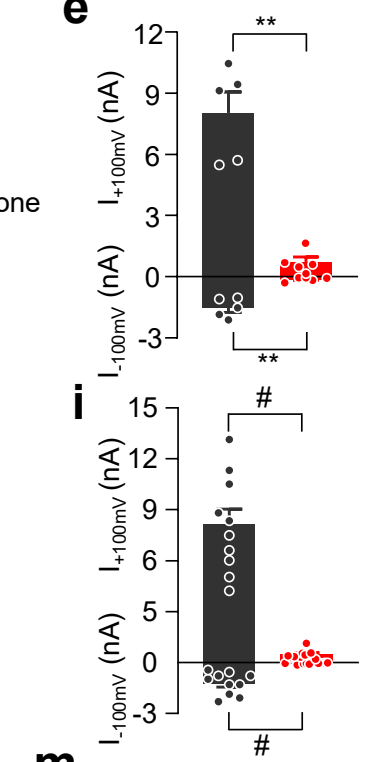

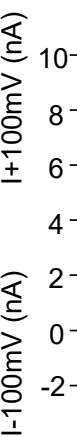

p

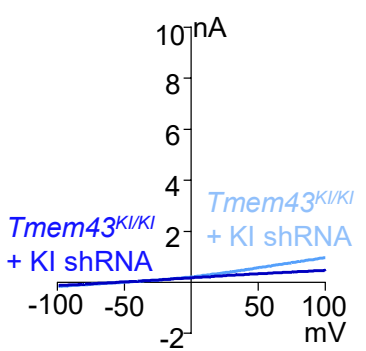

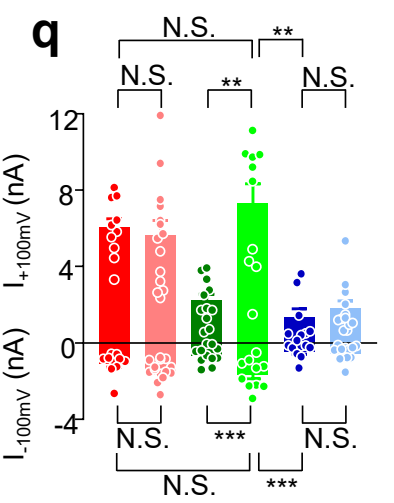


bioRxiv preprint doi: https://doi.org/10.1101/2020.07.27.222323; this version posted July 27, 2020. The copyright holder for this preprint (which Figure 7

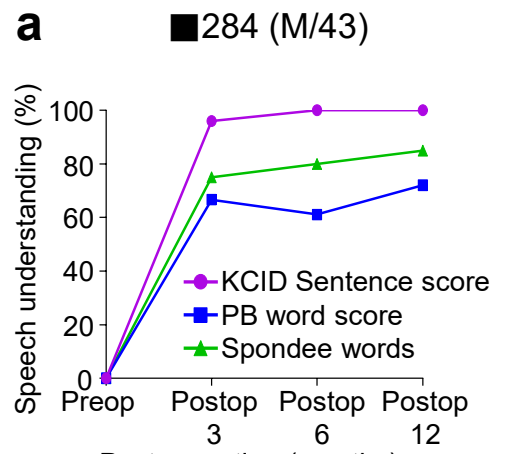

Post operation (months)

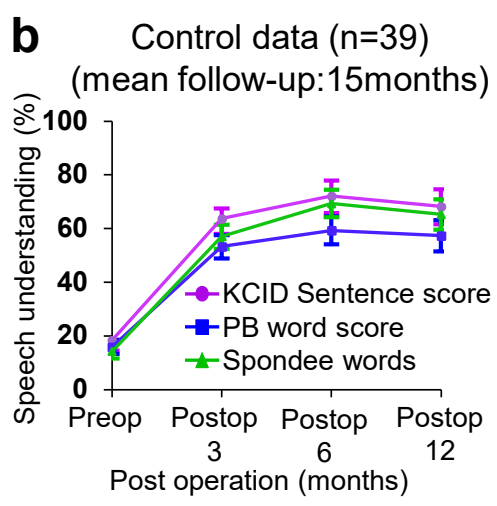

Post operation (months) d

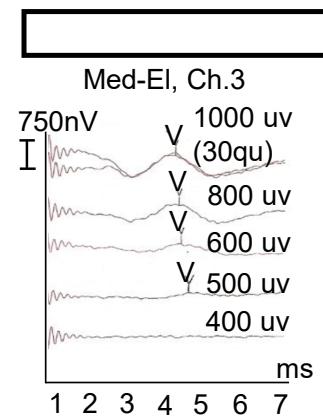

\section{e-ABR 0304 (F/53)}

Med-El, Ch.5

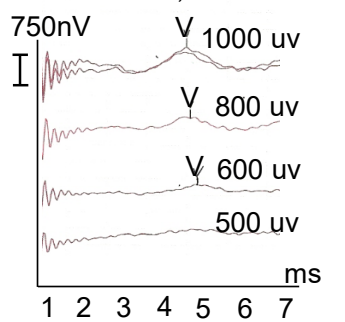

Med-El, Ch.8

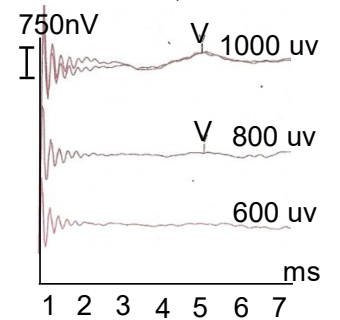

C

$0304(F / 53)$

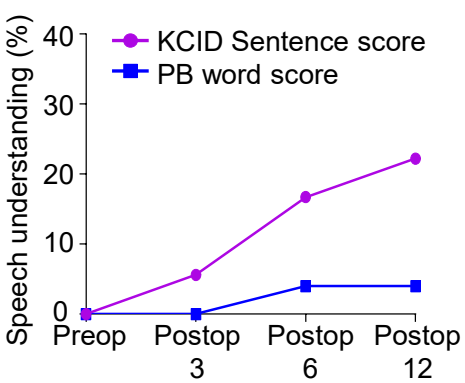

Post operation (months)

e

e-ABR (Postsynaptic ANSD)

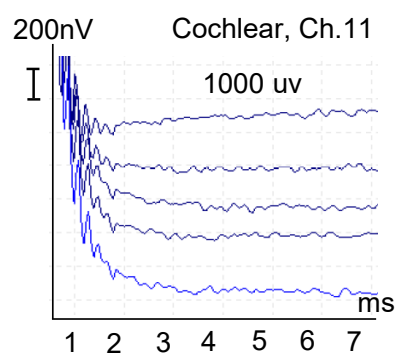


Table 1 | Nonsynonymous variants within the locus ${ }^{*}$ co-segregating with ANSD that survived filtering process after exome sequencing among 4 subjects from SB162

\begin{tabular}{|c|c|c|c|c|c|c|c|c|c|c|c|c|c|c|c|c|c|}
\hline Func & Gene & ExonicFunc & GenbankID & Position & Exon & Nucleotide & AA & Chr & Start & End & Ref & Alt & Global MAF & $\begin{array}{l}\text { KRGDB } \\
(\mathrm{N}=1722)\end{array}$ & PolyPhen-2 & SIFT & GERF++ \\
\hline \multicolumn{18}{|c|}{ Targeted exome sequencing after linkage analysis } \\
\hline exonic & TMEM43 & Stopgain SNV & NM_024334 & $\underset{2}{\operatorname{Region} \#}$ & exon12 & c.C1114T & p.R372X & chr3 & $14,183,206$ & $14,183,206$ & C & $\mathrm{T}$ & $\mathrm{T}=0.000008 / 1$ (ExAC) & ND & $\mathrm{NA}$ & $\mathrm{NA}$ & 4.83 \\
\hline exonic & FBLN2 & $\begin{array}{l}\text { nonsynonymous } \\
\text { SNV }\end{array}$ & NM_001998 & $\begin{array}{c}(3 p 25.1) \\
\text { Region \# } \\
2 * \\
2 . * \\
(3 p 25.1) \\
\end{array}$ & exon11 & c.G2551C & p.D851H & chr3 & $13,670,527$ & $13,670,527$ & G & $\mathrm{C}$ & $\begin{array}{c}\mathrm{C}=0.00007 / 84 \text { (EXAC) } \\
\mathrm{C}=0.0004 / 2 \\
(1000 \text { Genomes) }\end{array}$ & $\begin{array}{c}\mathrm{C}=0.005517 / 1 \\
9\end{array}$ & 0.893 & 0.04 & 4.23 \\
\hline \multicolumn{18}{|c|}{ Exome sequencing } \\
\hline exonic & KIAA1614 & nonsynonymous & NM_020950 & & exon5 & c.G2514T & p.E838D & chr1 & 180905559 & 180905559 & G & $\mathrm{T}$ & ND & ND & 0.002 & 0.39 & -5.05 \\
\hline exonic & АDСКз & $\begin{array}{l}\text { nonsynonymous } \\
\text { SNV }\end{array}$ & NM_020247 & & exon6 & c.С818T & p.A273V & chr1 & 227169815 & 227169815 & C & $\mathrm{T}$ & $\begin{array}{c}\mathrm{T}=0.00006 / 5 \text { (EXAC) } \\
\mathrm{T}=0.00000 / 1 \text { (GO-ES) } \\
\mathrm{T}=0.0001 / 4 \text { (TOPMED) }\end{array}$ & ND & 0.999 & 0.04 & 5.1 \\
\hline exonic & ТВСE & nonsynonymous & NM_001079515 & & exon9 & c.T794C & p.I265T & chr1 & 235599116 & 235599116 & $\mathrm{~T}$ & $\mathrm{C}$ & $\begin{array}{l}\mathrm{T}=0.0001 / 4 \text { (TOPMED) } \\
\mathrm{ND}\end{array}$ & ND & 0.01 & 0.62 & 4.61 \\
\hline exonic & PARD3B & $\begin{array}{c}\text { nonsynonymous } \\
\text { SNV }\end{array}$ & NM_057177 & & exon11 & c.A1594C & p.1532L & chr2 & 206023605 & 206023605 & A & C & ND & $\mathrm{ND}$ & 0.28 & 0.08 & -1.97 \\
\hline exonic & HDLBP & $\begin{array}{c}\text { nonsynonymous } \\
\text { SNV }\end{array}$ & NM_001243900 & & exon21 & c.G2728A & p.G910S & chr2 & 242176107 & 242176107 & C & $\mathrm{T}$ & $\begin{array}{c}\mathrm{T}=0.0000008 / 1 \text { (ExAC) } \\
\mathrm{T}=0.0002 / 1(1000 \\
\text { Genomes) }\end{array}$ & ND & 0.094 & 0.64 & 0.666 \\
\hline exonic & TMEM43 & stopgain SNV & NM_024334 & & exon12 & c.C1114T & p.R372X & chr3 & 14183206 & 14183206 & C & $\mathrm{T}$ & $\mathrm{T}=0.000008 / 1(\mathrm{ExAC})$ & ND & $\mathrm{NA}$ & $\mathrm{NA}$ & 4.83 \\
\hline exonic & DTX3L & nonsynonymous & NM_138287 & & exon1 & c.G83A & p. $528 \mathrm{~N}$ & chr3 & 122283356 & 122283356 & G & A & ND & ND & 0.454 & 0.03 & -9.39 \\
\hline exonic & KIAA1024L & $\begin{array}{l}\text { nonsynonymous } \\
\text { SNV }\end{array}$ & NM_001257308 & & exon1 & c.T160G & p. $554 \mathrm{~A}$ & chr5 & 129084043 & 129084043 & $\mathrm{~T}$ & G & ND & ND & $\mathrm{NA}$ & $\mathrm{NA}$ & 3.53 \\
\hline exonic & Р4HA2 & nonsynonymous & NM_001017973 & & exon2 & c.T80C & p. $.27 \mathrm{~T}$ & chr5 & 131554240 & 131554240 & A & G & ND & $\mathrm{G}=0.00029 / 1$ & 0.01 & 0.07 & 6.17 \\
\hline exonic & рокз & $\begin{array}{l}\text { nonsynonymous } \\
\text { SNV }\end{array}$ & NM_001144875 & & exon2 & c.С8T & p.P3L & chr5 & 176936534 & 176936534 & G & A & $\mathrm{ND}$ & $\mathrm{ND}$ & 0.991 & 0.03 & 4.47 \\
\hline exonic & MAML1 & $\begin{array}{l}\text { nonsynonymous } \\
\text { SNV }\end{array}$ & NM_014757 & & exon5 & c.C2338T & p.H780Y & chr5 & 179201165 & 179201165 & C & $\mathrm{T}$ & ND & $\mathrm{T}=0.00029 / 1$ & 0.916 & 0.31 & 4.94 \\
\hline exonic & ARID1B & $\begin{array}{c}\text { nonfram } \\
\text { inseshifift }\end{array}$ & NM_017519 & & $\begin{array}{l}\text { exon1,e } \\
\text { xon1 }\end{array}$ & $\begin{array}{l}\text { c.1029_1030i } \\
\text { nsGCG }\end{array}$ & p.A343deli & chr6 & 157100092 & 157100092 & - & $\underset{G}{G C}$ & ND & $\begin{array}{l}\text { InsGCG }=0.00 \\
1142 / 6\end{array}$ & $\mathrm{NA}$ & $\mathrm{NA}$ & -0.657 \\
\hline exonic & EPC1 & $\begin{array}{l}\text { nonsynonymous } \\
\text { SNV }\end{array}$ & NM_001272004 & & exon4 & c.T572C & p.I191T & chr10 & 32582010 & 32582010 & A & G & ND & $\mathrm{ND}$ & 0.999 & 0.07 & 6.04 \\
\hline exonic & CRYAB & $\begin{array}{c}\text { SNV } \\
\text { nonsynonymous } \\
\text { SNV }\end{array}$ & NM_001885 & & exon1 & c.T79A & p.F27I & chr11 & 111782370 & 111782370 & A & $\mathrm{T}$ & ND & $\mathrm{ND}$ & 0.002 & 0.35 & 2.56 \\
\hline exonic & INO80 & $\begin{array}{c}\text { nonsynonymous } \\
\text { SNV }\end{array}$ & NM_017553 & & exon6 & c.A623G & p.K208R & chr15 & 41379795 & 41379795 & $\mathrm{~T}$ & C & $\mathrm{C}=0.0001 / 17$ (ExAC) & $\mathrm{C}=0.0046457 /$ & 0.009 & 0.61 & 4.2 \\
\hline exonic & MYO5C & nonsynonymous & NM_018728 & & exon29 & c.A3620C & p.E1207A & chr15 & 52515748 & 52515748 & $\mathrm{~T}$ & G & ND & $\mathrm{ND}$ & 0.365 & 0.73 & 5.97 \\
\hline exonic & CILP & $\begin{array}{l}\text { nonsynonymous } \\
\text { SNV }\end{array}$ & NM_003613 & & exon5 & c.C448G & p.R150G & chr15 & 65497781 & 65497781 & G & $\mathrm{C}$ & ND & ND & 0.0 & 0.67 & 0.901 \\
\hline exonic & МАР2K1 & $\begin{array}{l}\text { nonsynonymous } \\
\text { SNV }\end{array}$ & NM_002755 & & exon10 & c.A1062C & p.Q354H & chr15 & 66782095 & 66782095 & A & c & $\mathrm{C}=0.00002 / 2$ (EXAC) & $\mathrm{C}=0.001161 / 4$ & 0.001 & 0.02 & -5.39 \\
\hline exonic & ZFPM1 & nonsynonymous & NM_153813 & & exon10 & c.C1645G & p.L549V & chr16 & 88600011 & 88600011 & C & G & ND & $\mathrm{G}=0.00029 / 1$ & 0.978 & 0.01 & 2.61 \\
\hline exonic & MIDN & $\begin{array}{c}\text { SNV } \\
\text { nonsynonymous } \\
\text { SNV }\end{array}$ & NM_177401 & & exon5 & c.C401T & p.S134L & chr19 & 1254182 & 1254182 & c & $\mathrm{T}$ & $\begin{array}{c}\mathrm{T}=0.000003 / 2 \text { (ExAC) } \\
\mathrm{T}=0.0002 / 5 \text { (TOPMED) }\end{array}$ & ND & 1 & 0 & 3.8 \\
\hline
\end{tabular}

${ }^{*}$ Locus: Region 1+ Region 2 (See Figure 1c inset); ${ }^{* *}$ Region \#1: Chr 3: 1,946,000 - 5,956,000; Region \#2: Chr 3:11,883,000-14,502,000; Ref: Reference sequence; Alt: Alternate sequence; ND: not detected; NA: not applicable. 
The candidate variants were firstly listed up if they were nonsynonymous variants in coding regions. Variants with a minor allele frequency less than $1 \%$ were then selected based on the Exome Sequencing Project 6500 (ESP6500), the 1000 Genome Project (1000G), The Exome Aggregation Consortium (ExAC), Trans-Omics for Precision Medicine (TOPMED) and our in-house database containing the exomes of 81 Korean individuals. Following the inheritance pattern, homozygous variants and compound heterozygote variants with sufficient read depths (>10X) and a genotype quality (>20) were selected commonly found in the

affected siblings. Finally, the variants which have none of clinical significance of dbSNP ID were finally identified. In silico prediction Algorithm: Polyphen-2 (http://genetics.bwh.harvard.edu/pph2/index shtml); SIFT (http://sift.jcvi.org/www/SIFT_chr_coords_submit.html); Conservation tools: GERP++ score in the UCSC Genome Browser (http://genome-asia.ucsc.edu/) ; ExAC, Exome Aggregation Consortium

(http://exac.broadinstitute.org/) ; 1000 Genomes (https://www.ncbi.nlm.nih.gov/variation/tools/1000genomes/) ; KRGDB, Korean Reference Genome DB (http://152.99.75.168/KRGDB/); TOPMED, Trans-Omics for Precision Medicine (https://www.nhlbiwgs.org). 